\title{
Target-induced clustering activates Trim-Away of pathogens and proteins
}

\author{
Jingwei Zeng ${ }^{1}$, Ana Filipa Santos ${ }^{2}$, Aamir S. Mukadam ${ }^{3}$, Mariana Osswald ${ }^{2}$, David A. \\ Jacques $^{4}$, Claire F. Dickson ${ }^{4}$, Stephen H. McLaughlin ${ }^{1}$, Christopher M. Johnson ${ }^{1}$, Leo \\ Kiss $^{1}$, Jakub Luptak ${ }^{1}$, Nadine Renner ${ }^{1}$, Marina Vaysburd ${ }^{1}$, William A. McEwan ${ }^{3,}{ }^{,}$, Eurico \\ Morais-de-Sá ${ }^{2,}$, , Dean Clift ${ }^{1,{ }^{*}}$, Leo C. James ${ }^{1,{ }^{*}}$ \\ ${ }^{1}$ Medical Research Council, Laboratory of Molecular Biology, Cambridge, UK \\ 2i3S - Instituto de Investigação e Inovação em Saúde and IBMC Instituto de Biologia Molecular e \\ Celular, Universidade do Porto, Porto, Portugal \\ ${ }^{3}$ UK Dementia Research Institute, Department of Clinical Neurosciences, University of \\ Cambridge, Cambridge, UK \\ ${ }^{4}$ EMBL Australia Node, Single Molecule Science, School of Medical Sciences, University of New \\ South Wales, Sydney, Australia
}

\begin{abstract}
Trim-Away is a recently developed technology that exploits off-the-shelf antibodies and the E3 RING ligase and cytosolic antibody receptor TRIM21 to carry out rapid protein depletion. How TRIM21 is catalytically activated upon target engagement, either during its normal immune function or when re-purposed for targeted protein degradation, is unknown. Here we show that a mechanism of target-induced clustering triggers intermolecular dimerisation of the RING domain, to switch on the ubiquitination activity of TRIM21 and induce virus neutralisation or drive Trim-Away. We harness this mechanism for selective degradation of disease-causing huntingtin protein containing long polyQ tracts, and expand the Trim-Away toolbox with highly active TRIM21-nanobody chimeras that can also be controlled optogenetically. This work provides a mechanism for cellular activation of TRIM RING ligases and has implications for targeted protein degradation technologies.
\end{abstract}

E3 ubiquitin ligases catalyse the final step in the ubiquitination reaction, which involves the transfer of ubiquitin from an E2 enzyme onto a target. There are more than 600 E3 ligases in the human genome, which regulate pathways involved in all aspects of eukaryotic biology.

\footnotetext{
*Correspondence: William McEwan (wm305@cam.ac.uk); Eurico Morais-de-Sá (eurico.sa@ibmc.up.pt); Dean Clift (dclift@ mrclmb.cam.ac.uk); Leo C. James (lcj@mrc-lmb.cam.ac.uk).

Author Contributions

D.C. and L.C.J. conceptualized the study; J.Z., M.O., W.A.M., E.M.S., D.C. and L.C.J. developed the methodology; J.Z., A.F.S., A.S.M., M.O., D.A.J., C.F.D., S.H.M., C.M.J., L.K., J.L., N.R., M.V., W.A.M., and D.C. performed the experiments; D.C. and L.C.J. prepared the manuscript; J.Z., A.F.S., M.O., W.A.M., E.M.S., D.C. and L.C.J. revised and edited the manuscript; W.A.M., E.M.S., D.C. and L.C.J. supervised the work; W.A.M., E.M.S and L.C.J. acquired funding.

Competing Interests

The authors declare no competing interests.
} 
Typically, E3 ligases have evolved to ubiquitinate a dedicated substrate or set of substrates. Protein binding domains control which substrates a particular E3 ligase can interact with, while post-translational modifications such as phosphorylation and neddylation add further layers of regulation to control E3 ligase activation and ensure specificity of the ubiquitin transfer reactions ${ }^{1}$. Sub-cellular localisation and tissue-specific expression provides an additional level regulation at the cell and organism level ${ }^{2,3}$.

Despite this apparent exquisite specificity, E3 ligases are increasingly being re-purposed by researchers to ubiquitinate non-canonical targets. In this approach, intermediary molecules such as chemical compounds, peptides, antibodies or engineered binding domains are designed to artificially re-direct E3 ligases to proteins of interest, with the aim of causing their degradation through the ubiquitin-proteasome system. This approach has huge potential as a protein knockdown research tool as well as an entirely new therapeutic modality ${ }^{4,5}$. However, precisely how E3 ligase activity is activated upon indirect recruitment to noncanonical targets remains unclear.

Interestingly, evolution has already produced an E3 ubiquitin ligase whose function is to mediate degradation of diverse targets via an intermediary molecule. TRIM21 is a member of the tripartite motif (TRIM) family of RING E3 ubiquitin ligases, but uniquely among TRIMs, and indeed all cytosolic proteins, TRIM21 possesses high-affinity antibody-binding activity $^{6}$. TRIM21 uses antibodies as intermediary molecules to direct a wide-range of targets for proteasomal degradation including viruses ${ }^{7-11}$, bacteria ${ }^{12,13}$ and proteopathic agents such as Tau ${ }^{14}$. TRIM21 underpins a system of intracellular immunity where the diversity of the body's antibody repertoire can be utilised in the cytosol to degrade invading pathogens ${ }^{15,16}$. This broad-spectrum targeting capability of TRIM21 has recently been exploited to develop Trim-Away, an easy to use and powerful method for acute and rapid degradation of endogenous cellular proteins. Trim-Away works by delivering antibodies to cells by electroporation or microinjection ${ }^{17,18}$. Because Trim-Away utilizes standard off-theshelf antibodies and no prior modification of proteins with tags or epitopes is required, this method has quickly been adopted to target a wide range of different proteins in various cell types and even model organisms.

One explanation for how TRIM21 degrades diverse targets via an intermediate is that it does not need to directly engage with or ubiquitinate its target. Instead, it may ubiquitinate itself and cause the entire TRIM21-antibody-target complex to be co-degraded. Consistent with this, all three complex components undergo degradation with identical kinetics during Trim-Away ${ }^{17}$. TRIM21 has also been shown to autoubiquitinate itself in vitro with an $\mathrm{N}$-terminal monoubiquitin via Ube2W, which is then extended into an anchored K63-linked chain by Ube $2 \mathrm{~N}^{19}$. A specific RING mutation that inhibits Ube2N recruitment, but not the promiscuous E2 Ube2D, prevents TRIM21 function in cells ${ }^{20}$. However, whether TRIM21 ubiquitinates itself during target engagement within the cell is unknown. Many RING E3 ligases have been observed to undergo autoubiquitination in vitro, yet substrate ubiquitination is required for their cellular function ${ }^{21}$. It is also unusual to drive degradation using K63 chains, as K48 are the canonical proteasomal degradation linkage. K63 chains have been shown to mediate both proteasome recruitment ${ }^{22-24}$ and substrate degradation ${ }^{25}$, but some studies suggest that degradation only occurs because K63 chains serve as a 
scaffold for branched K48-chain formation ${ }^{26}$. The anti-HIV restriction factor TRIM5, another member of the TRIM family, shares many of the same ubiquitination characteristics as TRIM21. TRIM5 also autoubiquitinates its $\mathrm{N}$-terminus with Ube2W and then recruits Ube2N to form an anchored K63-ubiquitin chain ${ }^{27}$. Depleting these E2 $\mathrm{s}^{27}$ or mutating the TRIM5 RING to prevent E2 interaction ${ }^{28}$ inhibits TRIM5-mediated degradation. The unusual involvement of K63-chains in TRIM5- and TRIM21-mediated degradation may represent a more widespread feature of the TRIM protein family and one that distinguishes it from other RING ligases.

TRIMs represent the largest family of RING E3s yet how they are activated and regulated is poorly understood. Their unusual domain architecture likely plays a key role in both processes. Most TRIMs are believed to form dimers in which a central antiparallel coiledcoil positions each RING domain at either end of the molecule. As many RINGs are only active as dimers, this may keep TRIMs inactive ${ }^{29}$. Several mechanisms have been proposed to explain how RING dimerization takes place to allow TRIM-mediated ubiquitination. It has been suggested that TRIM25 RINGs may dimerise intramolecularly because the B Box domains fold back over the coiled-coil, placing the two RINGs together at the centre of the molecule ${ }^{30}$. Alternatively, TRIM25 RINGs may dimerise intermolecularly when two TRIM25 dimers stack end-to-end ${ }^{31}$. This may be facilitated by TRIM25 binding to RNA, which could bring multiple TRIM 25 molecules into proximity ${ }^{31,32}$. TRIM higher order assembly has been studied in most detail for TRIM5. Unusually for a RING ligase, TRIM5 is constitutively active, continuously undergoing rapid autoubiquitination and turnover by the proteasome ${ }^{33}$. This is thought to occur because the B Box domain drives self-assembly of oligomers, bringing together multiple RING domains ${ }^{34}$, which may facilitate RING dimerisation $^{35}$. During HIV restriction, TRIM5 forms a regular hexagonal lattice around the hexagonal capsid lattice, leading to an arrangement of three RINGs at each vertex ${ }^{36,37}$. This facilitates a 'two-plus-one' mechanism of ubiquitination in which a RING dimer ubiquitinates the orphan $\mathrm{RING}^{28}$.

Neither constitutive activation nor B Box-mediated assembly is likely to be shared by all TRIM proteins. TRIM25 does not turnover or require its B Boxes to oligomerise with RNA in phase-separated droplets ${ }^{31}$. TRIM21, although continuously expressed in most tissues, also does not constitutively ubiquitinate or degrade itself. Previously we have shown that the TRIM21 B Box inhibits RING autoubiquitination in vitro by competing for E2 Ub binding ${ }^{38}$. However, it is unclear how B Box inhibition is relieved. Phosphorylation of RING residue $\mathrm{S} 80$ helps to displace the B Box and promotes immune signalling but it is not required for TRIM21-mediated degradation ${ }^{38}$. This suggests that target engagement must somehow activate TRIM21. How this can occur even though target binding occurs indirectly via antibodies is unknown. In this study we show that a process of intermolecular clustering induced by antibody-complexed targets drives the dimerisation of RING domains from neighbouring TRIM21 molecules and triggers K63-linked autoubiquitination and degradation. We exploit the TRIM21 clustering mechanism to selectively degrade diseasecausing huntingtin protein, and develop new tools for targeted protein degradation using TRIM21. 


\section{Results}

\section{TRIM21 assembles K63-linked Ub chains on itself upon target engagement}

We first investigated whether target engagement is the trigger for TRIM21 enzymatic activity. We infected cells overexpressing His-tagged ubiquitin with adenovirus (AdV5) alone or in the presence of wild-type anti-AdV5 hexon antibody (9C12) or a mutant antibody that cannot bind TRIM21 [9C12(H433A) ${ }^{39}$. TRIM21 underwent polyubquitination in the presence of AdV5+9C12 antibody but not AdV5 alone. Polyubiquitination was lost with the $9 \mathrm{C} 12(\mathrm{H} 433 \mathrm{~A})$ antibody, confirming that target engagement by TRIM21 is required (Extended Data Fig. 1a). In cells expressing His-tagged TRIM21 at endogenous levels and with endogenous ubiquitin, we observed a similar pattern of ubiquitination that was reversed with the deubiquitinase USP2 (Extended Data Fig. 1b-e).

To determine whether TRIM21 itself is activated, or if it merely becomes a target for another ubiquitin ligase, we introduced mutations into the TRIM21 RING domain (Extended Data Fig. 1f) that impair E2 Ub binding and reduce both Trim-Away and AdV5 neutralisation ${ }^{20}$. Of these mutations, E12R and E13A substantially reduced polyubiquitination (Extended Data Fig. 1a,g). This indicates that TRIM21 is catalytically activated upon target engagement. To address which ubiquitin chain types TRIM21 modifies itself with upon target engagement in cells, we expressed different ubiquitin mutants. Expression of K48R, which prevents K48-chain formation, did not impact TRIM21 polyubiquitination. Consistent with this, no polyubiquitination was seen in cells expressing a ubiquitin mutant that forms K48-linked chains only. In contrast, K63R substantially reduced TRIM21 polyubiquitination whilst cells expressing only K63 maintained chain formation (Extended Data Fig. 1h). This suggests that TRIM21 builds K63 chains on itself upon target engagement in cells.

\section{RING dimerisation is required for TRIM21 activation}

To investigate whether TRIM21 RING dimerisation is required for function, we mutated two residues at the RING dimer interface (Fig. 1a) that are required for RING dimerisation ${ }^{38}$. The M72E mutation abolished TRIM21's ability to degrade target protein using TrimAway (Fig. 1b) and reduced TRIM21 autoubiquitination upon infection with AdV5+9C12 (Extended Data Fig. 2a-c). Previously, we showed that monomeric TRIM21 RING domain possesses catalytic activity but is constitutively inhibited by the B Box ${ }^{38}$. However, comparing RING (T21R), RING-B Box (T21RB) or RING-linker-RING (T21RLR; two RINGs in a single polypeptide) constructs revealed that enforcing RING dimerisation significantly increases TRIM21 catalytic activity, as measured by polyubiquitin synthesis and ubiquitin discharge from Ube2N Ub (Fig. 1c-e). This increase in activity of the RLR construct required the M10 dimerisation residue (Extended Data Fig. 2d). Thus, RING dimerisation both increases TRIM21 catalysis in vitro and is necessary for TRIM21 activity in cells.

TRIM21 is a multi-domain protein containing RING, B Box, coiled-coil and PRYSPRY domains (Extended Data Fig. 3a). Using SEC-MALS and isothermal calorimetry, we found that the coiled-coil (T21CC235; residues 129-235) from TRIM21 undergoes monomerdimer exchange but forms no higher order multimers (Extended Data Fig. 3b,c). This result 
was confirmed by analytical ultracentrifugation using a T21RBCC construct containing residues 1-235 (Extended Data Fig. 3d). Small angle x-ray scattering (SAXS) data on T21CC235 and T21RBCC (Extended Data Fig. 3e-g) revealed that the TRIM21 dimer exists as an elongated antiparallel coiled-coil with the two copies of the RING domain held apart by at least $150 \AA$ (Fig. 1f; Supplementary Note 1). This arrangement was maintained in full length TRIM21 bound to IgG Fc (Fig 1g; Extended Data Fig. 3h-j; Supplementary Note 1), suggesting that antibody binding does not induce large scale conformational changes in TRIM21 that could facilitate intramolecular RING dimerisation.

\section{RING dimerisation is induced by recruitment of multiple TRIM21 molecules to a target}

How can TRIM21 RING domains dimerise when they are held apart as monomers by an elongated coiled-coil? A potential mechanism could be dimerisation of RING domains between neighbouring TRIM21 dimers, for example as a result of TRIM21 clustering on the surface of an antibody-coated virion (Fig. 1h). This clustering mechanism predicts that recruitment of multiple TRIM21 molecules to the target is required for TRIM21 activation. We tested this prediction using a virus neutralisation assay in which the infectivity of adenoviral particles can be accurately quantified ${ }^{7}$. We used saturating concentrations of 9C12 antibody, in which 200 antibodies bind per virion ${ }^{39}$, but varied ratios of 9C12(WT) and 9C12(H433A) mutant, which lacks an intact TRIM21 binding site. Full neutralisation was abrogated when $9 \mathrm{C} 12(\mathrm{H} 433 \mathrm{~A})$ reached $>75 \%$. For one natural $\log$ neutralization, $\sim 12 \%$ of WT antibodies were required; by assuming WT and H433A antibodies were randomly distributed between viruses, we calculate that $\sim 24$ WT out of the 200 antibodies bound to AdV5 at saturation were required for neutralisation (Fig. 1i). Thus, efficient neutralisation only occurs when multiple TRIM21 molecules are recruited to the target.

Introducing the dimerisation mutants M72E or M10E significantly increased the amount of antibody required to initiate neutralisation and rendered TRIM21 unable to activate NFKB signalling (Fig. 1j,k). Furthermore, mutations N71D and R67A at the RING dimer:Ub interface (Extended Data Fig. 1f) also led to an increase in the critical threshold of antibodies required for neutralisation (Extended Data Fig. 2e-g). These mutations also reduced T21RLR polyubiquitination in vitro and rendered TRIM21 unable to activate NFKB signalling or degrade target using Trim-Away (Extended Data Fig. 2d,h,i). Altogether, these data indicate that catalytically-active RING dimers are formed upon recruitment of multiple TRIM21 molecules to the target.

\section{Trim-Away requires recruitment of multiple TRIM21 to target}

The above data support a model in which TRIM21 becomes catalytically-active when RING dimerisation is induced by the clustering of multiple TRIM21 molecules on an antibody-coated target (Fig. 1h). We tested this model by using an engineered target protein containing a repeated epitope sequence. To this end, we designed a series of monomeric EGFP (mEGFP) reporter constructs with four copies of the 10 amino acid c-myc tag at the $\mathrm{N}$-terminus. We serially introduced the point mutations L4A and I5A, which ablate binding by the monoclonal anti-myc antibody $9 \mathrm{E} 10^{40}$. This antibody cannot induce antigen crosslinking owing to its atypical binding mode wherein both Fab domains contribute to antigen binding in a 'clasping' manner ${ }^{41}$. This resulted in a series of constructs with between zero 
and four anti-myc antibody binding sites per mEGFP (Fig. 2a). Upon electroporation of anti-myc antibody, we only observed efficient degradation of mEGFP with 4 active anti-myc binding sites (Fig. 2b-d). Degradation of the 4myc construct was antibody dose-dependent, whereas there was no loss of mEGFP containing only 3 anti-myc binding sites at any antibody concentration (Fig. 2e). Finally, to confirm that antibody binding takes place to the 2 myc construct, but that a critical number of antibodies has not been reached, we co-electroporated anti-IgG polyclonal antibody alongside anti-myc and observed efficient degradation (Fig. 2f).

To confirm these results using a target protein without epitope tags, we mixed purified GFP together with either monoclonal or polyclonal anti-GFP antibodies and determined the molecular weight of the resulting protein complexes by mass photometry ${ }^{42,43}$. In the absence of GFP, all the antibodies used gave a peak of approximately $150 \mathrm{kDa}$ (Fig. $2 \mathrm{~g}-\mathrm{i}$, grey lines), consistent with the predicted size of a single IgG. For both a single monoclonal antibody (9F9.F9), and a mix of two monoclonals $(7.1+13.1)$, the addition of GFP resulted in a peak shift to approximately $180-240 \mathrm{kDa}$, consistent with one or two GFP molecules ( 27kDa) bound by just a single antibody (Fig. $2 \mathrm{~g}, \mathrm{~h}$, black lines). However, for the polyclonal antibody (ab6556), the addition of GFP resulted in the appearance of a peak at $330 \mathrm{kDa}$ (Fig. 2i, black line), indicating two antibodies binding a single GFP molecule. We next tested which GFP:antibody complexes could trigger TRIM21 activation, and thus GFP degradation, inside cells. Only electroporation of polyclonal anti-GFP antibody (ab6556) resulted in efficient degradation of mEGFP (Fig. 2j). TRIM21 was co-depleted together with mEGFP when using the polyclonal antibody (Fig. 2j), consistent with TRIM21 activation only in this condition. All anti-GFP antibodies used were capable of binding GFP inside living cells (Extended Data Fig. 4). Altogether, these data suggest that protein degradation requires the recruitment of multiple TRIM21 molecules to the target.

\section{The nature of the target dictates TRIM21 activation}

As Trim-Away of endogenous cellular proteins can be performed efficiently with monoclonal antibodies ${ }^{17}$, we hypothesised that the nature of the target itself could lead to the clustering of multiple TRIM21 molecules in close proximity and induce TRIM21 activation. To investigate this further, we compared the degradation of different GFP-fusion proteins (Fig. 3a-c) using an anti-GFP nanobody-Fc chimera (vhhGFP4-Fc) ${ }^{17}$, which binds a single epitope on GFP ${ }^{44}$. We co-expressed vhhGFP4-Fc with either monomeric EGFP (mEGFP) or mEGFP fused to oligomeric scaffolds Histones (H2B-mEGFP) or Centrosomes (mEGFP-PACT). As with electroporation of monoclonal antibody (Fig. 2j), expression of vhhGFP4-Fc did not cause degradation of mEGFP (Fig. 3d,e). However, there was efficient degradation mEGFP when fused to the oligomeric scaffolds, as visualised by loss of nuclear GFP fluorescence for H2B-mEGFP (Fig. 3f,g) and loss of centrosomal puncta for mEGFPPACT (Fig. 3h,i). Introducing the H433A mutation into the Fc region of vhhGFP4-Fc abolished degradation of mEGFP-PACT or H2B-mEGFP, confirming TRIM21 dependence (Fig. 3f-i). Expression of the vhhGFP4-Fc construct in cells where EGFP has been knockedin to a single allele of Caveolin- $1^{45}$, which is highly oligomeric ${ }^{46}$, led to similar TRIM21dependent degradation (Fig. 3j-1). Altogether these data suggest that the nature of the target dictates TRIM21 activation during Trim-Away with single epitope binders. 
We next asked if we could take advantage of target-induced TRIM21 activation to selectively degrade proteins that cause disease. Huntington's disease is caused by expansion of the polyglutamine (polyQ) tract at the N-terminus of huntingtin (Htt) protein. Tracts $<36 \mathrm{Q}$ do not cause disease, whereas tracts $>39 \mathrm{Q}$ are certain to cause disease, with severity increasing with polyQ tract length ${ }^{47}$. The monoclonal anti-polyglutamine antibody $3 \mathrm{~B} 5 \mathrm{H} 10$ binds to a 13Q epitope, which means that increasing numbers of 3B5H10 antibodies can bind as the Htt polyQ tract length increases ${ }^{48}$. We hypothesised that the 3B5H10 antibody would recruit more TRIM21 molecules to long polyQ tracts than short, resulting in TRIM21 clustering and degradation of only disease-causing huntingtin (Fig. 4a). To test this, we expressed mEGFP-tagged huntingtin exon 1 with polyQ tracts increasing in 13Q increments up to 78Q and monitored GFP fluorescence following electroporation of 3B5H10 antibody. Strikingly, the degree of degradation caused by the 3B5H10 antibody correlated with polyQ tract length. Tracts $>39$ Q were efficiently degraded, whereas tracts $<39$ Q were spared from degradation, with tracts of exactly 39Q showing an intermediate level of degradation (Fig. 4b,c). Thus, target-induced TRIM21 activation could be exploited therapeutically for selective degradation of disease proteins without the need for selective binders.

\section{Molecular clustering is sufficient to activate TRIM21}

The above data suggest that TRIM21 activation is triggered by the clustering of multiple TRIM21 molecules in close proximity, either by multiple antibodies bound to the target or by the oligomeric nature of the target itself. To test if molecular clustering is sufficient to activate TRIM21, we developed an assay that would allow us to induce TRIM21 clustering independently of a target. To this end, we exploited the properties of engineered variants of the plant photoreceptor, Cryptochrome 2 (CRY2), which undergo clustering upon exposure to blue light ${ }^{49-51}$. We generated constructs with CRY2 fused to TRIM21 (Fig. 5a) and compared their behaviour in cells. Exposure of cells to blue light resulted in the rapid formation of cytoplasmic puncta for both mRFP-CRY2 and mRFP-CRY2-TRIM21 constructs, consistent with CRY2 clustering. Strikingly, live microscopy revealed that these clusters rapidly disappeared in cells expressing mRFP-CRY2-TRIM21, but not in cells expressing mRFP-CRY2. The loss of CRY2-TRIM21 puncta was fully prevented by the addition of the proteasome inhibitor MG132, suggesting that this was due to proteasomal degradation (Fig 5b,c; Supplementary Video 1), which was confirmed by western blotting (Fig. 5d). Introducing mutations that abolish E2 Ub binding (E13A) and RING dimerisation (M10E/M72E) both prevented clustering-induced TRIM21 degradation (Fig. 5d). Together this shows that TRIM21 is normally catalytically inert, but that upon molecular clustering active RING dimers form and drive degradation.

\section{Expanding the Trim-Away toolbox}

We investigated if we could exploit clustering-induced TRIM21 activation to achieve optogenetic control of Trim-Away. To this end, we generated an optoTrim-Away construct containing TRIM21 fused to both the anti-GFP nanobody and light-controllable CRY2 domain (vhhGFP4-RFP-CRY2-TRIM21; Fig. 6a). We expressed this construct in cells together with the intended target of Trim-Away, GFP-tagged protein kinase C (GFP-aPKC), which behaves as a monomer in non-polarized cells ${ }^{52}$. At the onset of blue light stimulation, both proteins were expressed and distributed diffusely in the cytoplasm, suggesting that 
recruitment of TRIM21 to GFP-aPKC was insufficient to cause degradation. However, within minutes of blue light stimulation GFP-aPKC formed clusters together with vhhGFP4RFP-CRY2-TRIM21 that were rapidly degraded (Fig. 6b,c). Degradation of GFP-aPKC was TRIM21-dependent, because co-clustering of aPKC with RFP-CRY2-vhhGFP4 in the absence of TRIM21 did not lead to degradation (Fig. 6d,e).

Our data suggests that intermolecular clustering activates TRIM21 by driving RING dimerisation. In support of this model, we found that just the TRIM21 RING domain alone was sufficient to induce proteasomal degradation upon molecular clustering (Fig. 6f). We exploited this finding to engineer a minimal degrader molecule that combines the catalytic activity of the TRIM21 RING together with a targeting domain in a single polypeptide chain (mini-Trim-Away; T21R-vhhGFP4; Fig. 6g). Strikingly, expression of T21R-vhhGFP4 caused rapid and stable proteasomal degradation of targets Caveolin-1-EGFP and H2BmEGFP (Extended Data Fig. 5).

A key advantage of the original Trim-Away method is the direct delivery of protein reagents (whole antibodies) to the cell, which allows rapid target protein degradation even in primary cells that are sensitive to classical nucleotide delivery approaches ${ }^{17}$. We found that the T21R-vhhGFP4 protein expressed exceptionally well in bacteria and could be purified using a simple two-step protocol to give high yields and purity (Fig. 6h; Methods). Electroporation of this protein into Caveolin-1-EGFP knock-in cells resulted in dose-dependent degradation of Caveolin-1-EGFP beginning immediately after protein delivery (Fig. 6i). The small size of the T21R-vhh protein reagent $(27 \mathrm{kDa})$ makes it ideally suited to target proteins sequestered in the nucleus that cannot be accessed by whole antibodies ${ }^{17}$. To test this, we electroporated the protein into primary mouse embryonic fibroblasts (MEFs) isolated from H2B-GFP transgenic mice. Remarkably, H2B-GFP was degraded within 1h of T21RvhhGFP4 protein electroporation (Fig. 6j). Protein delivery outperformed mRNA delivery in primary MEFs (Fig. 6j), likely because primary cells are sensitive to exogenous nucleotides, highlighting the advantage of the T21R-vhh protein reagent for Trim-Away in primary cells.

\section{Discussion}

Our data suggest the following mechanism for TRIM21 activation and regulation (Fig. 7): TRIM21 is expressed as a catalytically inactive dimer, in which the two copies of its RING domain are located at either end of an elongated antiparallel coiled-coil. Separating the two RINGs in each TRIM21 molecule reduces activity with E2 Ub, which requires a pre-formed RING dimer for efficient catalysis. Ubiquitination activity is triggered by targetinduced clustering of TRIM21, for instance by a viral particle with several antibodies bound, which promotes intermolecular RING dimerisation and makes E2 Ub binding energetically favourable. Once activated, TRIM21 transfers a mono-ubiquitin onto its own N-terminus, which is extended into an anchored K63-linked chain, and both TRIM21 and the target are degraded by the proteasome. While the data suggests that K63-ubiquitination is essential for activity, it remains unclear whether additional modifications, such as branched ubiquitin chains, are involved. Ectopically expressed TRIM21 is ubiquitinated first with K63-chains and then with K48-chains, suggesting branched ubiquitin chains can form ${ }^{19}$. 
This mechanism of clustering-induced activation has important implications for targeted protein degradation with TRIM21, as monomeric proteins targeted with a monoclonal antibody cannot be degraded. For some Trim-Away applications this may be a limitation, however this can be overcome by simply co-delivering a polyclonal secondary antibody together with the primary targeting antibody. We also show how the requirement for TRIM21 clustering for degradation can be exploited (Fig. 7). Firstly, by fusing TRIM21 to the light-inducible clustering domain CRY2, degradation of monomeric proteins can be controlled optogenetically (optoTrim-Away). This may be particularly useful in model organisms, where spatial control of Trim-Away may allow tissue-specific depletion. Secondly, we show that target-induced TRIM21 clustering can be exploited for selective degradation of disease-causing huntingtin protein. Such an approach may be applicable in other cases where disease is caused by expansion of a poly-amino acid repeat sequence, or aggregation of an intracellular protein that is normally monomeric, both of which are common in neurodegenerative disorders ${ }^{53,54}$.

We have further exploited our new understanding of TRIM21 mechanism to engineer a minimal degrader molecule (miniTrim-Away) that allows specific protein depletion without antibodies by fusing the TRIM21 RING domain with a nanobody. Genetically encoding Trim-Away into a single polypeptide may be useful in biological systems where whole antibody delivery is not possible and paves the way for therapeutic delivery via gene therapy vectors in vivo. Alternatively, delivery of TRIM21 RING-nanobody chimeras as protein reagents circumvents both the necessity for endogenous TRIM21 expression or any genetic manipulation and may be particularly useful for protein depletion experiments in primary cells. This work expands the Trim-Away technology toolbox beyond the use of whole antibodies and allows more flexibility when designing protein depletion experiments utilising TRIM21. In addition to Trim-Away there are a growing number of methods available for acutely controlling endogenous protein levels ${ }^{4,5}$. However, our work here on TRIM21 provides a mechanism to explain how an E3 ligase can be re-directed to degrade diverse non-canonical targets. Further work will be crucial to allow researchers to make an informed choice of which E3 ligase to choose for targeted degradation of their protein of interest, either as a research tool or therapeutic.

Our construction of a CRY2 light-inducible clustering system demonstrates that TRIM21 achieves degradation by acting both as enzyme and substrate. This is consistent with observations that TRIM21 modifies itself with a polyubiquitin chain in vitro ${ }^{19}$ and when complexed with antibody-coated virus in cells (here). This use of autoubiquitination has important functional consequences: by ubiquitinating itself, TRIM21 eliminates the requirement for a correctly orientated lysine residue within the antibody or target. This may explain how TRIM21 can target rapidly diverging pathogens during infection and be exploited to degrade any intracellular protein using Trim-Away technology. TRIM5 also ubiquitinates itself and is co-degraded alongside the HIV capsid during its restriction of viral infection $^{28,55}$. However, it remains unclear whether targets of TRIM5 or TRIM21 rely solely on being complexed with the ubiquitinated ligase to be degraded or if they are also modified during processing. Future work is also needed to determine whether other TRIMs operate in a similar way. 
Most TRIMs have in common a central coiled-coil domain, suggesting that they all employ spatial separation of their RINGs to regulate ubiquitination activity. Our data suggests that RING dimers form between neighbouring TRIM21 molecules when clustered together upon target engagement (Fig. 7). We propose that a mechanism of clustering-induced RING dimerisation is shared by other TRIM family members, although modes of clustering may differ. For TRIM5, a combination of B Box-induced self-assembly and recruitment to the hexagonal HIV capsid lattice brings three RING domains into close proximity to activate ubiquitination $^{28,34-37}$. For TRIM25 intermolecular RING dimerisation may be facilitated by RNA binding leading to either RNA-mediated TRIM25 higher order assembly, or simply inducing high enough concentrations of TRIM 25 molecules within phase-separated RNA granules for RING dimerisation to occur ${ }^{31,32}$. Clustering may also be important to activate non-TRIM ligases, for instance it has been shown to activate the Nedd4 family of HECT domain E3 ligases ${ }^{56}$ and small molecule-induced clustering of BCL6 makes it a better substrate for the RING E3 ligase SIAH $1^{57}$. Thus, target-induced clustering may be a general mechanism of activation utilised across the ubiquitin ligase family.

\section{Methods}

Mice

The experiments involving mice have been approved by the Medical Research Council Animal Welfare and Ethical Review Body and the UK Home Office under project licence number PCF3F9520. All mice were maintained in a specific pathogen-free environment according to UK Home Office regulations. H2B-GFP mice (CAG:H2B-EGFP) were obtained from The Jackson Laboratory (strain 006069).

\section{Cell culture}

HEK293T (ATCC) and NIH3T3-Caveolin-1-EGFP ${ }^{45}$ were cultured in DMEM medium (Gibco; 31966021) supplemented with 10\% Calf Serum and penicillin-streptomycin. RPE-1 cells (ATCC) were cultured in DMEM/F-12 medium (Gibco; 10565018) supplemented with $10 \%$ Calf Serum and penicillin-streptomycin. All cells were grown at $37^{\circ} \mathrm{C}$ in a $5 \% \mathrm{CO}_{2}$ humidified atmosphere and regularly checked to be mycoplasma-free. The sex of NIH3T3 cells is male. The sex of HEK293T and RPE-1 cells is female. Drosophila S2DGRC cells (Drosophila Genomics Resource Centre) were cultured at $25^{\circ} \mathrm{C}$ in Schneider's Insect medium (Sigma-Aldrich, St. Louis, MO, USA) supplemented with 10\% Calf serum. For primary mouse embryonic fibroblasts (MEFs) isolation, uteri isolated from 13.5-daypregnant mice were washed with PBS. The head and visceral tissues were removed from isolated embryos. The remaining bodies were washed in fresh PBS, transferred into $3 \mathrm{ml}$ of $0.25 \%$ trypsin, $1 \mathrm{mM}$ EDTA and minced using 2 scalpels. After 20 min incubation at $37^{\circ} \mathrm{C}$ cell suspension was mixed with $25 \mathrm{ml}$ of DMEM medium supplemented with $10 \%$ Calf Serum and strained through $40 \mu \mathrm{m}$ cell strainer. Cells were collected by centrifugation (200 $\times \mathrm{g}$ for $5 \mathrm{~min}$ at $4^{\circ} \mathrm{C}$ ), resuspended in fresh medium and seeded in T75 flask (passage 1). In this study, we used MEFs within three passages to avoid replicative senescence. 


\section{Plasmids}

A full list of plasmids used in this study and details of plasmid construction can be found in Supplementary Note 2. For stable expression of TRIM21 at endogenous levels, a native TRIM21 promoter-driven lentiviral vector (pT21MPP) was used ${ }^{58}$. For in vitro mRNA transcription, constructs were cloned into PEMHE $^{59}$, which contains UTR and polyA sequences for optimal mRNA stability and translation. For all TRIM21 constructs, sequences encoding amino acids 1-85 were used for the RING domain (T21R), amino acids 1-129 for RING-Box (T21RB), amino acids 132-235 for Coiled-Coil (T21CC235) and amino acids 1-235 for RING-Box-Coiled-Coil (T21RBCC).

\section{TRIM21 knockout cell lines}

HEK293T TRIM21 knockout cells were described previously ${ }^{38}$. RPE-1 TRIM21 knockout cells were generated using the Alt-R CRISPR-Cas9 system from Integrated DNA technologies (IDT) with a custom-designed crRNA sequence (ATGCTCACAGGCTCCACGAA). Guide RNA in the form of crRNA-tracrRNA duplex was assembled with recombinant Cas9 protein (IDT \#1081060) and electroporated into RPE-1 cells together with Alt-R Cas9 Electroporation Enhancer (IDT \#1075915). Two days post-electroporation cells were plated one cell per well in 96 well plates and single cell clones screened by western blotting for TRIM21 protein. A single clone was chosen that contained no detectable TRIM21 protein and confirmed TRIM21 knockout phenotype in a Trim-Away assay.

\section{Stable cell lines}

Stable cell lines generated and used in this manuscript are detailed in Supplementary Note 2. HEK293T-mCherry-hTRIM21 cells were described previously ${ }^{17}$. Lentivirus particles were collected from HEK293T supernatant 3 days after co-transfection of lentiviral plasmid constructs together with HIV-1 GagPol expresser pcRV1 (a gift from Dr. Stuart Neil) and pMD2G, a gift from Didier Trono (Addgene plasmid \#12259). Supernatant was filtered at $0.45 \mu \mathrm{m}$ before storage at $-80^{\circ} \mathrm{C}$. For stable expression of TRIM21 at endogenous levels, HEK293T TRIM21 KO cells were transduced with lentivirus particles at multiplicity $\sim 0.1$ transducing units per cell and selected using puromycin at $2.5 \mu \mathrm{g} / \mathrm{ml}$ from 48 hours posttransduction $^{58}$. For stable expression of H2B-mEGFP-FKBP, RPE-1 cells were transduced at multiplicity $\sim 0.1$ transducing units per cell, sorted into single cells, and a single cell clone selected after screening for GFP expression by flow cytometry.

\section{Electroporation}

Electroporation was performed using the Neon ${ }^{\circledR}$ Transfection System (Thermo Fisher). Cells were washed with PBS and resuspended in Buffer R at a concentration of $8 \times 10^{7}$ cells $\mathrm{ml}^{-1}$. For each electroporation reaction $8 \times 10^{5}$ cells $(10.5 \mu \mathrm{l})$ were mixed with $2 \mu \mathrm{l}$ of antibody (typically $0.5 \mathrm{mg} / \mathrm{ml}$ ) or mRNA (typically $0.5 \mu \mathrm{M}$ ) or protein to be delivered. The mixture was taken up into a $10 \mu \mathrm{l} \mathrm{Neon}{ }^{\circledR}$ Pipette Tip, electroporated at 1400V, $20 \mathrm{~ms}, 2$ pulses and transferred to media without antibiotics. 


\section{Ubiquitin and TRIM21 pull-down assays}

Approximately $4 \times 10^{5}$ cells were transfected with $250 \mathrm{ng}$ of pMT107-octa-6 His-ubiquitin or $200 \mathrm{ng}$ of pcDNA3-2x-6His-ubiquitin. Media was changed 24h post-transfection. At $48 \mathrm{~h}$ post-transfection, media was replaced with serum-free $\mathrm{CO}_{2}$ independent medium (Gibco; 18045088) and the cells were chilled on ice for 30 min before infection with AdV5 or AdV5-9C12 complex for a further $30 \mathrm{~min}$ on ice. $\triangle \mathrm{E} 1 \Delta \mathrm{E} 3$ AdV5-GFP (ViraQuest) was diluted 1:4 in PBS and mixed 1:1 with either $20 \mu \mathrm{g} / \mathrm{ml}$ of humanised $9 \mathrm{C} 12 \mathrm{IgG} 3 \mathrm{~b}$ or PBS and incubated for $1 \mathrm{hr}$ at $20 \mathrm{C}$ to allow for complex formation. Following infection, cells were incubated at $37^{\circ} \mathrm{C}$ for $30 \mathrm{~min}$, washed twice with PBS and collected by scraping. Cells pellets were collected by centrifugation and flash frozen in liquid nitrogen. For the denaturing His-Ubiquitin pulldowns, cell pellets were lysed with $500 \mu \mathrm{l}$ of buffer A containing $6 \mathrm{M}$ Guanidinium chloride, $5 \mathrm{mM}$ imidazole $100 \mathrm{mM}$ sodium phosphate buffer $\mathrm{pH} 7.4$, mixed by vortexing and sonicated in ultrasonic water bath for $2 \mathrm{~min}$ (10s on/off) with amplitude to $40 \%$ to shear the DNA. $20 \mu \mathrm{l}$ of buffer equilibrated Ni-NTA bead slurry (50\% bead) were added to each sample and incubated for at least $3 \mathrm{hrs}$ on a rotating wheel at $4 \mathrm{C}$. Beads were washed 2x with buffer A, 2x with buffer B (buffer A and buffer C mixed 1:3) and $2 x$ with buffer $C$ ( $20 \mathrm{mM}$ Tris, $20 \mathrm{mM}$ imidazole $\mathrm{pH}$ 6.8). Supernatant was removed using $30 \mathrm{G}$ needle and beads boiled for $5 \mathrm{~min}$ in $10 \mu \mathrm{l}$ of $2 \mathrm{X} \mathrm{LDS}+100 \mathrm{mM}$ DTT + $300 \mathrm{mM}$ imidazole for SDS-PAGE analysis. For denaturing TRIM21-His pulldown, lysis buffer containing 4M Urea, $5 \mathrm{mM}$ imidazole $50 \mathrm{mM}$ Tris $\mathrm{pH} 7.4$ was used and where indicated, the washed Ni-NTA beads were treated with USP2 (Boston Biochem; K-400) before immunoblotting.

\section{Adenovirus neutralisation assay}

HEK293T Cells were plated at a density of $5 \times 10^{4}$ cells/well in $0.5 \mathrm{ml}$ of media in $24-$ well plates and were allowed to attach overnight. $\triangle \mathrm{E} 1 \Delta \mathrm{E} 3 \mathrm{AdV} 5-\mathrm{GFP}$ (Viraquest) was diluted 1:1250 in PBS and mixed 1:1 with 9C12 antibody at the indicated concentrations and incubated for 1 hour at $20^{\circ} \mathrm{C}$ to allow for complex formation. For estimating the stoichiometry of TRIM21 for neutralisation, 9C12(H433A) antibody was mixed at the indicated ratio with $9 \mathrm{C} 12(\mathrm{WT})$ at $1 \mathrm{mg} / \mathrm{ml}$ before addition to AdV5-GFP particles. For the infection, $10 \mu \mathrm{l}$ of the virus or virus-antibody complex was added to each well. Cells were harvested by trypsinisation at 16-20 hrs post infection and evaluated for GFP expression on a BD LSRFortessa cell analyser (BD Biosciences). The results were analysed using FlowJo software (FlowJo LLC) and relative infection was calculated using the method described previously ${ }^{7}$.

\section{NFKB signalling assay}

Approximately $3 \times 10^{5}$ cells were transfected with 200 ng pGL4.32 NF- $\kappa$ B luciferase plasmid (Promega). Cells were incubated for six-hours at $37^{\circ} \mathrm{C}$ before reseeding at a density of $1 \times 10^{4}$ per well in 96-well plates (Corning CellBIND; 3340) and allowed to attach overnight. $\triangle \mathrm{E} 1 \triangle \mathrm{E} 3$ AdV5-GFP (ViraQuest) was diluted 1:3 in PBS and mixed 1:1 with either $20 \mu \mathrm{g} / \mathrm{ml}$ of humanised $9 \mathrm{C} 12 \mathrm{IgG} 3 \mathrm{~b}$ or PBS and incubated for $1 \mathrm{hr}$ at $20^{\circ} \mathrm{C}$ to allow for complex formation. $5 \mu \mathrm{l}$ of the virus or virus-antibody complex was added to each well in triplicates and allowed to incubate for 6 hrs before the cells were lysed with $50 \mu \mathrm{l} /$ well 
of steadylite plus luciferase reagent (Perkin Elmer; 6066751). The luciferase activity was quantified using a BMG PHERAstar FS plate reader.

\section{Light-induced CRY2 clustering}

Drosophila S2 cells were transiently transfected with Hsp70-promotor-controlled optogenetic constructs, seeded on glass bottom-dishes (MatTek Corporation, Ashland, MA, USA) coated with poly-lysine and between 2-5 days post-transfection expression induced by incubation at $37^{\circ} \mathrm{C}$ for $40 \mathrm{~min}$ at least $5 \mathrm{~h}$ prior to cell imaging. After induction, cells were kept in the dark and manipulated under a 593nm LED light (Super Bright LEDs, St. Louis, MO, USA). For proteasome inhibition, culture medium was supplemented with 20 $\mu \mathrm{M}$ MG132 at least $1 \mathrm{~h}$ before live imaging. The $470 \mathrm{~nm}$ filter of a Nikon Ti motorised inverted epifluorescence widefield microscope (Nikon, Japan) was used to induce CRY2 clustering, whereas the $555 \mathrm{~nm}$ filter was used to image RFP. For RPE-1 cells, following electroporation of mRNA, cells were incubated overnight. The following day, cells were shifted to $\mathrm{CO}_{2}$-independent medium (Gibco; 18045088) supplemented with $10 \%$ Calf Serum and Glutamax (Gibco; 35050061) with either DMSO or $20 \mu \mathrm{M}$ MG132 before being exposed to blue light (wavelength of 450-500 nm) from a Dark Reader transilluminator (Clare Chemical). Cells were harvested for immunoblot analysis after 3 hours of exposure to blue light. The washed cell pellets were lysed directly in $60 \mu \mathrm{l}$ of 1x LDS sample buffer, sonicated in a water bath sonicator for $5 \mathrm{~min}\left(30 \mathrm{~s} \mathrm{on} / \mathrm{off}, 4^{\circ} \mathrm{C}\right)$ and boiled at $95^{\circ} \mathrm{C}$ for 10 minutes before loading on to NuPAGE 4-12\% Bis-Tris gels (Thermo Fisher).

\section{Microscopy}

Live imaging of mammalian cells was performed using the IncuCyte S3 live cell analysis system (Sartorius) housed within a $37^{\circ} \mathrm{C}, 5 \% \mathrm{CO}_{2}$ humidified incubator. Live imaging of Drosophila $\mathrm{S} 2$ cells was performed using a Nikon Ti motorised inverted epifluorescence widefield microscope (Nikon, Japan), equipped with a Lumencor SpectraX light engine and external emission filter wheel, and an Andor iXon888 camera (Andor, UK), with a PL APO LAMBDA 60X OIL/1,4 Oil WD 0,13mm objective, controlled by NIS elements 5.0 (Nikon, Japan) software. Z-stacks were acquired as 7 serial sections $1.5 \mu \mathrm{m}$ apart. For fixed cells, images were acquired with a Leica SP8 microscope equipped with a 63x C-Apochromat 1.2 NA oil immersion objective.

\section{Measurement of fluorescence in live cells}

To quantify RFP and GFP degradation in S2 cells, images were captured just before blue light exposure and in 1-minute (Fig. 5b,c) or 2-minute (Fig. 6b-e) intervals from blue light exposure onwards. Using ImageJ/FIJI software ${ }^{60}$, the mean pixel intensity was measured within regions of interest (ROIs) that were manually tracked to include the complete area of each individual cell in which clustering occurred. Fluorescence intensity was quantified in average-intensity Z-projections covering all imaged planes and the signal was also measured in 3 non-transfected cells in the same sample, which were used for background subtraction. Fluorescence intensity over time is shown as the ratio between each timepoint and the second timepoint after light-induction. To quantify GFP fluorescence in live mammalian cells, images were acquired and analysed using the IncuCyte live cell analysis system (Sartorius). Within the IncuCyte software, the integrated density (the product of the area 
and mean intensity) for GFP fluorescence was normalised to total cell area (phase) for each image.

\section{Immunofluorescence}

Cells were fixed for $30 \mathrm{~min}$ at $37^{\circ} \mathrm{C}$ in $100 \mathrm{mM}$ HEPES ( $\mathrm{pH} 7$; titrated with $\mathrm{KOH}$ ), $50 \mathrm{mM}$ EGTA (pH 7; titrated with $\mathrm{KOH}$ ), $2 \%$ formaldehyde (methanol free) and $0.2 \%$ Triton X-100 and incubated in blocking buffer (PBS, 3\% BSA and $0.1 \%$ Triton X-100) for $1 \mathrm{~h}$ at room temp prior to antibody incubations. Antibodies and dilutions (in blocking buffer) used for immunofluorescence (IF) are detailed in Supplementary Note 2. DNA was stained with $5 \mathrm{mg}$ $\mathrm{ml}^{-1}$ Hoechst 33342 (Molecular Probes H3570). To measure mEGFP fluorescence, Image $\mathrm{J}$ software was used to segment images in the mEGFP channel and measure mean mEGFP intensity within the segmented region. H2B-mEGFP-positive nuclei and mEGFP-PACT foci, as well as total nuclei (Hoechst), were manually counted using the Image $\mathbf{J}$ cell counter tool.

\section{Immunoblotting}

Samples were run on NuPAGE 4-12\% Bis-Tris gels (Thermo Fisher) and transferred onto nitrocellulose membrane. Membranes were incubated in blocking buffer (PBS, $0.1 \%$ Tween $20,5 \%$ milk) for $1 \mathrm{~h}$ at room temp prior to incubation with antibodies. Antibodies and dilutions (in blocking buffer) used for immunoblotting (IB) are detailed in Supplementary Note 2. HRP-coupled antibodies were detected by enhanced chemiluminescence (Amersham, GE Healthcare) and X-ray films. IRDye-coupled antibodies were detected using LI-COR Odyssey CLx imaging system.

\section{Protein Purification}

Ubiquitin, Ube1, Ube2N(K92R), T21R (amino acids 1-85) and T21RB (amino acids 1-129) were produced as described previously ${ }^{19,20,38}$. The T21RLR and T21RBCC (amino acids 1-235) proteins were produced using the same protocol as $T 21 \mathrm{RB}^{38}$. To generate full length TRIM21 protein (T21FL), MBP-tagged TRIM21 (pFastBac-MBP-TRIM21) was expressed in SF9 insect cells in Lonza Insect-XPRESS according to standard protocols (Lonza). Cleared cell lysates were prepared by sonication of cell pellets in $50 \mathrm{mM}$ Tris $\mathrm{pH} \mathrm{8,} 200$ $\mathrm{mM} \mathrm{NaCl}, 1 \mathrm{mM}$ DTT, $10 \mu \mathrm{M} \mathrm{ZnCl}_{2}, 15 \%$ (vol/vol) BugBuster (Novagen) and cOmplete protease inhibitors (Roche), followed by centrifugation $16,000 \times \mathrm{g}$ for $30 \mathrm{~min}$. Lysates were loaded onto Amylose resin and the protein eluted with $10 \mathrm{mM}$ Maltose. MBP was cleaved from the eluted protein with TEV protease, and the protein was passed through a HiLoad 16/600 Superdex 200 PG size exclusion column using an ÄKTA pure purification system (GE Healthcare). Peak fractions were pooled and concentrated using Amicon Ultra-4 centrifugal filter units (Millipore). Proteins MBP-T21CC235 (containing MBP fused to TRIM21 residues 129-235) and T21CC235 (containing TRIM21 residues 129-235) were expressed in Escherichia coli C41(DE3) cells in 2TY medium and lysed and purified by affinity (NiNTA- or Amylose-resin, as appropriate) and size exclusion chromatography (SEC) as above, except the MBP tag was retained on MBP-T21CC235.

To generate the TRIM21-nanobody fusion protein, pOPTH-T21R-vhhGFP4 was transformed into E. coli $\mathrm{C} 41$ (DE3) and TRIM21-nanobody protein expressed using autoinduction media (ZYP-5052) for 20 hours at $30^{\circ} \mathrm{C}$. Cleared cell lysates were prepared 
by sonication of cell pellets in $50 \mathrm{mM}$ Tris $\mathrm{pH} 8,300 \mathrm{mM} \mathrm{NaCl}, 2 \mathrm{mM}$ DTT, $10 \mathrm{mM}$ Imidazole $\mathrm{pH} 8,15 \%$ (vol/vol) BugBuster (Novagen) and cOmplete protease inhibitors (Roche), followed by centrifugation $16,000 \times \mathrm{g}$ for $30 \mathrm{~min}$. Lysates were loaded onto Ni-NTA agarose (Qiagen), and protein eluted with $400 \mathrm{mM}$ Imidazole. Eluted protein was passed through a HiLoad 10/300 Superdex 75 GL size exclusion column using an ÄKTA pure purification system (GE Healthcare). Peak fractions were pooled and concentrated using Amicon Ultra-4 centrifugal filter units (Millipore). For electroporation into cells, proteins were dialysed in PBS for $2 \times 2$ hours using Slide-A-Lyzer ${ }^{\mathrm{TM}}$ Dialysis Cassettes (Thermo Fisher). Protein aliquots were frozen and stored at $-80^{\circ} \mathrm{C}$ until use.

\section{Ubiquitin discharge assay}

Ube2N(K92R) was loaded with ubiquitin by mixing $40 \mu \mathrm{M}$ of the E2 with $1 \mu \mathrm{M}$ Ube1, $0.37 \mathrm{mM} \mathrm{Ub}$ and $3 \mathrm{mM}$ ATP in $50 \mathrm{mM}$ HEPES pH 7.5, $150 \mathrm{mM} \mathrm{NaCl}, 20 \mathrm{mM} \mathrm{MgCl}_{2}$, and incubating the reaction at $37^{\circ} \mathrm{C}$ for $30 \mathrm{~min}$. The reaction was transferred to ice and used immediately. To observe E3 mediated discharge of ubiquitin, $2 \mu \mathrm{M}$ ubiquitin-loaded E2 was mixed with $2.5 \mu \mathrm{M}$ Ube2V2 and $1.5 \mu \mathrm{M}$ TRIM21 protein constructs in $50 \mathrm{mM}$ HEPES pH 7.5, $150 \mathrm{mM} \mathrm{NaCl}, 20 \mathrm{mM} \mathrm{MgCl} 2,50 \mathrm{mM}$ L-lysine. Samples were taken at timepoints post-mixing and reactions stopped by adding LDS sample buffer at $4^{\circ} \mathrm{C}$. The samples were boiled, resolved by SDS-PAGE and observed by immunoblot using rabbit anti-Ube2N (Bio-Rad, AHP974, 1:1000).

\section{Ubiquitin chain formation assay}

Reactions were carried out in $50 \mathrm{mM}$ Tris at $\mathrm{pH} 8,2.5 \mathrm{mM} \mathrm{MgCl} 2,0.5 \mathrm{mM}$ DTT with $0.2 \mathrm{mM}$ Ub, $2 \mathrm{mM}$ ATP, $1 \mu \mathrm{M}$ Ube1, $0.5 \mu \mathrm{M}$ Ube2N, $0.5 \mu \mathrm{M}$ Ube2V2 and $1.5 \mu \mathrm{M}$ TRIM21 protein constructs. The reaction was started upon incubation at $37^{\circ} \mathrm{C}$ for the time points indicated in the figures. The reaction was stopped via the addition of LDS sample buffer at $4^{\circ} \mathrm{C}$, followed by boiling at $90^{\circ} \mathrm{C}$ for $2 \mathrm{~min}$. The reactions were resolved by SDS-PAGE and ubiquitin detected by immunoblot using anti-ubiquitin-HRP (P4D1; Santa Cruz Biotechnology sc8017-HRP, 1:1000).

\section{Isothermal Titration Calorimetry (ITC)}

ITC dilution experiments were performed using an iTC200 titration calorimeter (Malvern Panalytical). T21CC235 was carefully dialysed into 20mM TRIS, pH 8, 100mM NaCl buffer at diluted to a concentration of $200 \mathrm{uM}$. This was titrated into the same dialysis buffer in the ITC cell using twenty $2 \mu \mathrm{L}$ injections. The resultant endothermic heat effects were integrated using Origin software supplied with the calorimeter.

\section{SEC-MALS}

Size exclusion chromatography coupled to multi angle light scattering (SEC-MALS) measurements were performed using a Wyatt Heleos II light scattering instrument coupled to a Wyatt Optilab rEX online refractive index detector (Wyatt Technology). Samples of T21CC235 (100uL) were resolved on a Superdex S-200 10/300 analytical gel filtration column (GE Healthcare) running at $0.5 \mathrm{ml} / \mathrm{min}$ in $20 \mathrm{mM}$ Tris $\mathrm{pH} 8,250 \mathrm{mM} \mathrm{NaCl}$ buffer before passing through the light scattering and refractive index detectors in a standard SEC-

Nat Struct Mol Biol. Author manuscript; available in PMC 2021 November 03. 
MALS format. Protein concentration was determined from the excess differential refractive index based on $0.186 \mathrm{RI}$ increment for $1 \mathrm{~g} / \mathrm{ml}$ protein solution. The concentration and the observed scattered intensity at each point in chromatogram were used to calculate the molar mass from the intercept of the Debye plot using Zimm's model as implemented in Wyatt's ASTRA software.

\section{Analytical ultracentrifugation (AUC)}

T21RBCC at approximately $0.5 \mathrm{mg} / \mathrm{mL}$ in $20 \mathrm{mM}$ Tris- $\mathrm{HCl}, \mathrm{pH}$ 7.5, $150 \mathrm{mM} \mathrm{NaCl}, 1 \mathrm{mM}$ DTT was loaded into $12 \mathrm{~mm}$ 2-sector cells, placed in an An50Ti rotor and centrifugated $50,000 \mathrm{rpm}$ at $10^{\circ} \mathrm{C}$ using an Optima XL-I analytical ultracentrifuge (Beckman). The data were analysed in SEDFIT $16.1 \mathrm{c}^{61}$ using a c(s) distribution model. The partial-specific volumes (v-bar), density and viscosity of the buffer were calculated using Sednterp (Dr Thomas Laue, University of New Hampshire). Data were plotted with the program GUSSI $^{62}$.

\section{Small Angle X-ray Scattering (SAXS)}

SAXS data $(\mathrm{I}(\mathrm{q})$ vs $\mathrm{q}$, where $\mathrm{q}=(4 \pi \sin \theta) / \lambda$, where $\lambda=1.24 \AA(10 \mathrm{kEV}), 2 \theta=$ scattering angle), were collected at the beam line P12 of the EMBL at the Petra-III storage ring (DESY, Hamburg). Samples were collected at 277 Kelvin using an EMBL/ESRF new generation automated sample changer (1.5 mm sample capillary). Samples were measured while flowing through the capillary with scattering intensities recorded using $50 \mathrm{~ms}$ exposure times (20 frames total) on a 2D photon counting Pilatus 2M pixel X-ray detector (Dectris), maintaining a fixed sample to detector distance of $3.1 \mathrm{~m}$ (q-range $0.008-0.47 \AA^{-1}$ ). For processing details, see Supplementary Note 1.

\section{Mass Photometry}

GFP:antibody mixtures were prepared in $300 \mathrm{mM} \mathrm{NaCl}, 50 \mathrm{mM}$ Tris $\mathrm{pH} 8$ and $1 \mathrm{mM}$ DTT and incubated on ice prior to loading onto the Refeyn One ${ }^{\mathrm{MP}}$ instrument (Refyn Ltd.) according to manufacturer's instructions. Data were processed and analysed using Discover MP v1.2.4 (Refeyn Ltd.). Events were then normalised with a calibration curve and the resulting data exported and processed in GraphPad Prism.

\section{Statistical Analysis}

Average (mean), standard deviation (s.d.), standard error of the mean (s.e.m) and statistical significance based on Student's $t$-test (two-tailed) and one- or two-way ANOVA were calculated in Microsoft Excel or Graphpad Prism. Significance are represented with labels

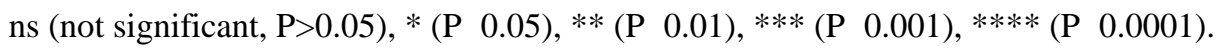




\section{Extended Data}
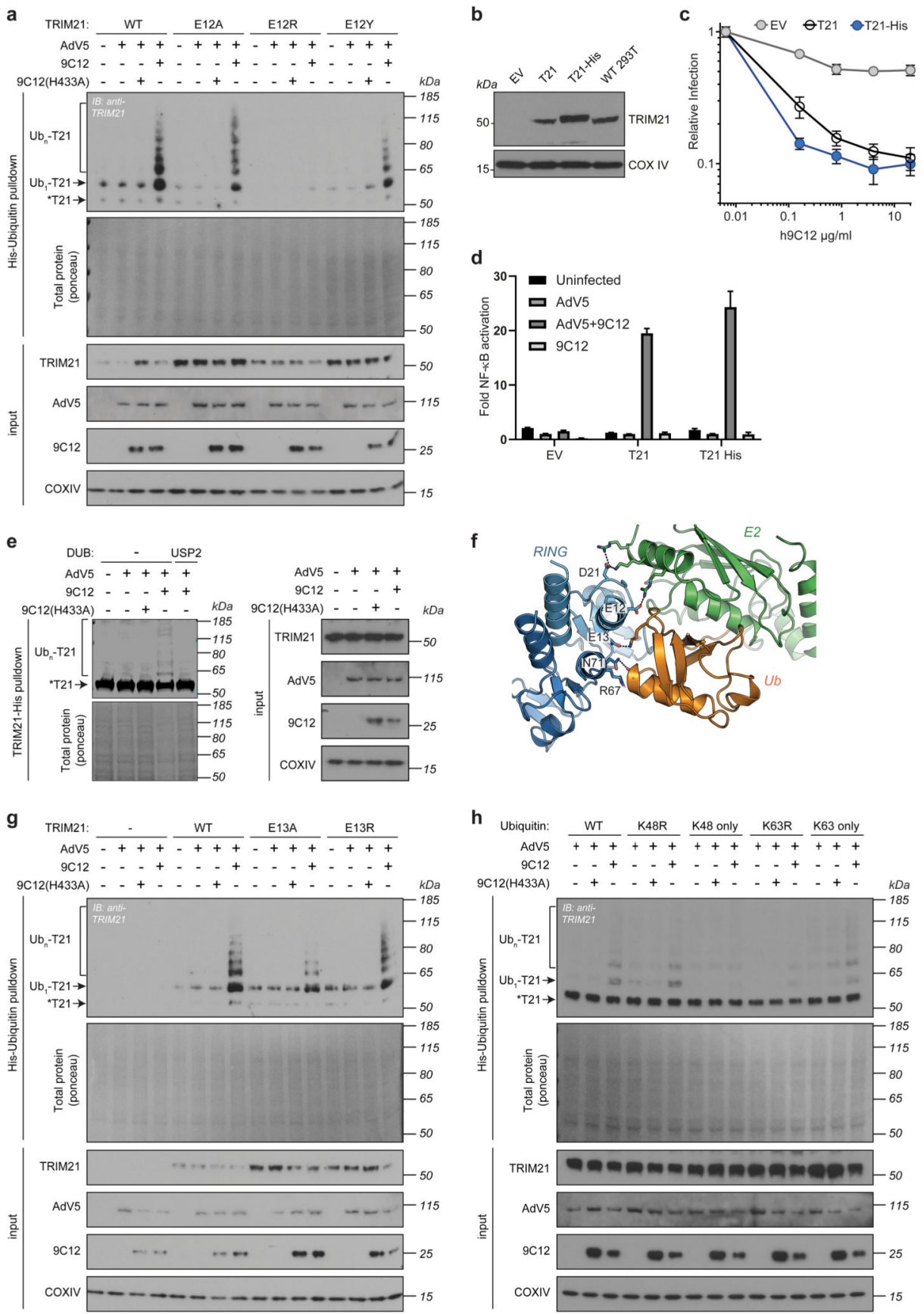

Extended Data Fig. 1.

Nat Struct Mol Biol. Author manuscript; available in PMC 2021 November 03. 
a

b
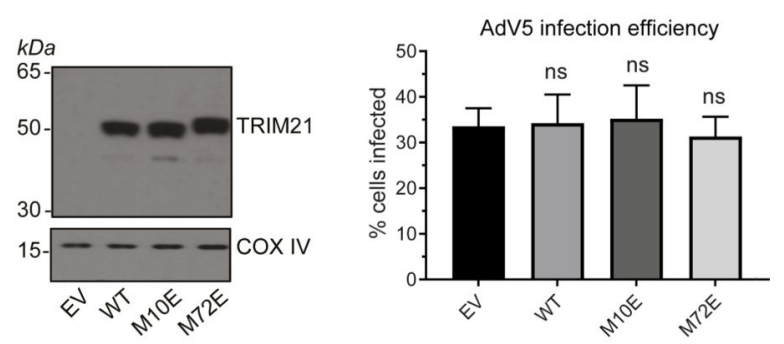

d T21RLR $\begin{array}{r}\text { T21RLR } \\ \text { M10E/M10 }\end{array}$

(min) $\overline{0152040} \overline{0152040} \frac{\mathrm{N} 52040}{\mathrm{kDa}}$
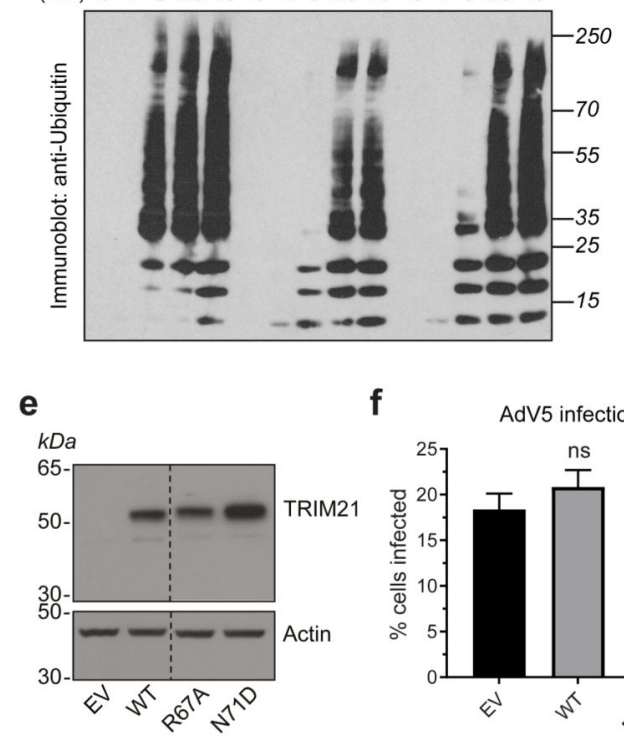

f AdV5 infection efficiency

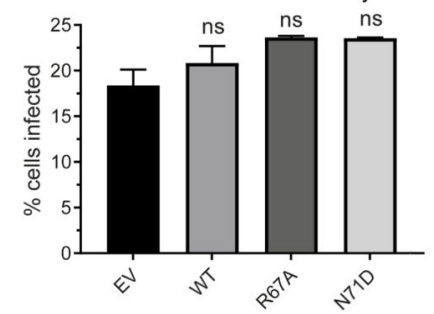

g

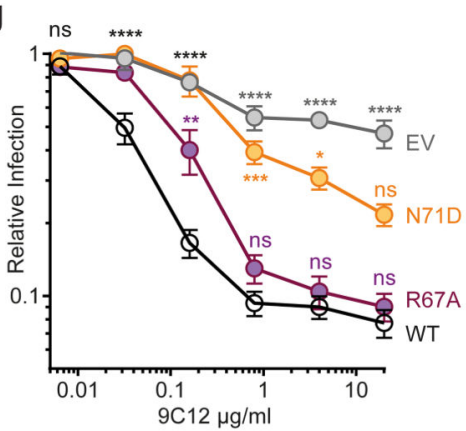

h

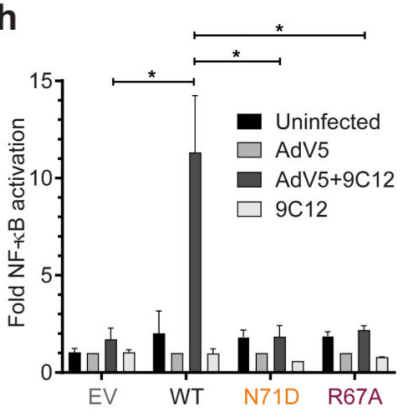

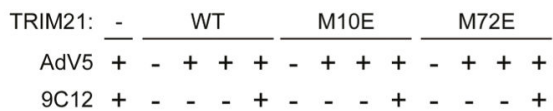
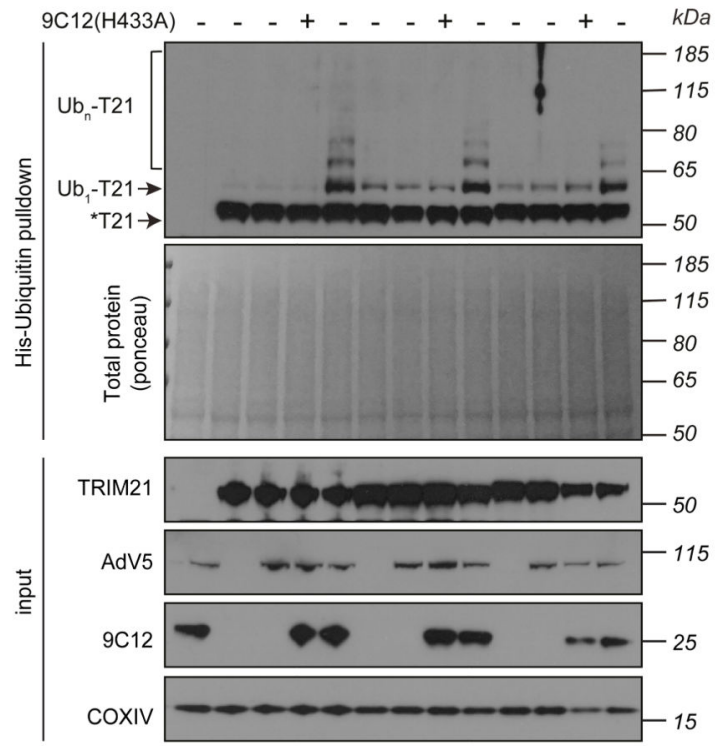

Extended Data Fig. 2.

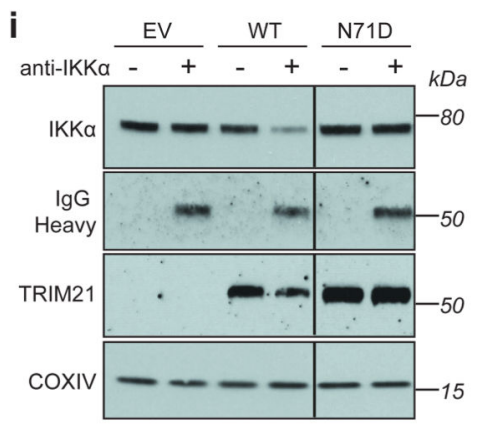

Nat Struct Mol Biol. Author manuscript; available in PMC 2021 November 03. 
a Human TRIM21 protein

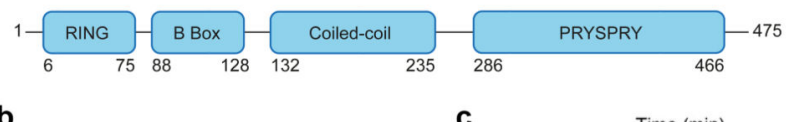

b

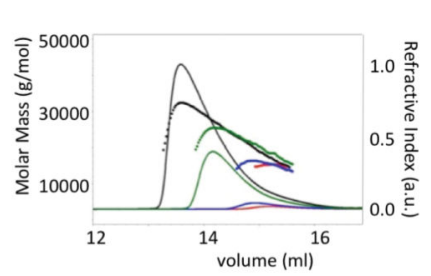

d
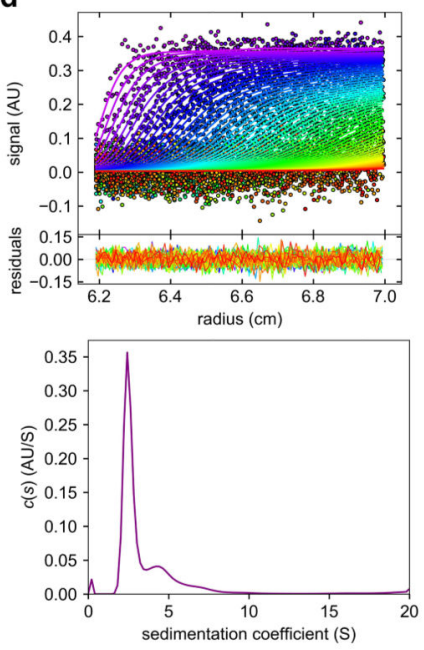

C

Time $(\min )$

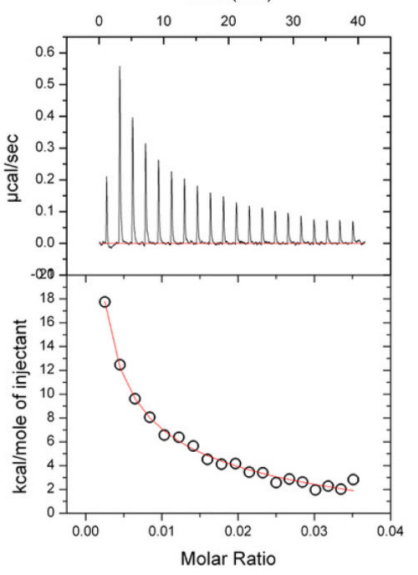

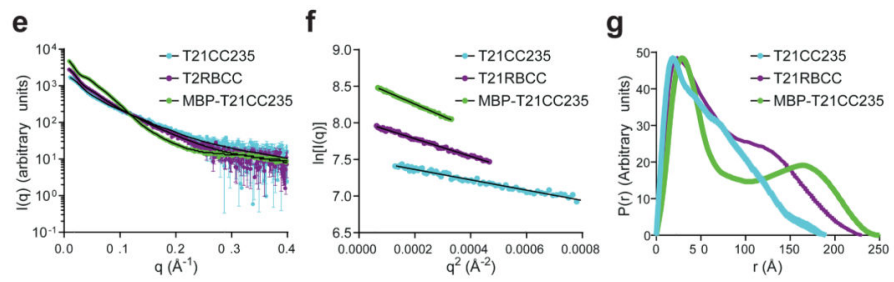
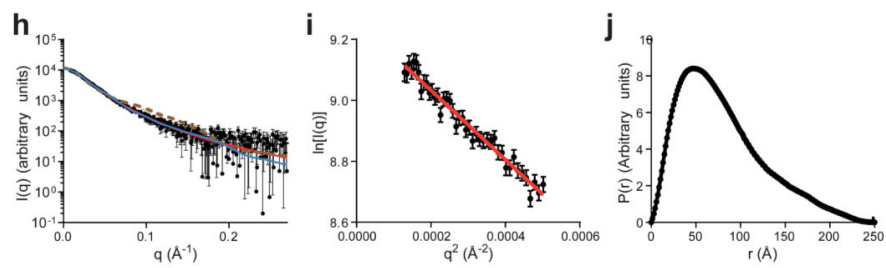

Extended Data Fig. 3. 


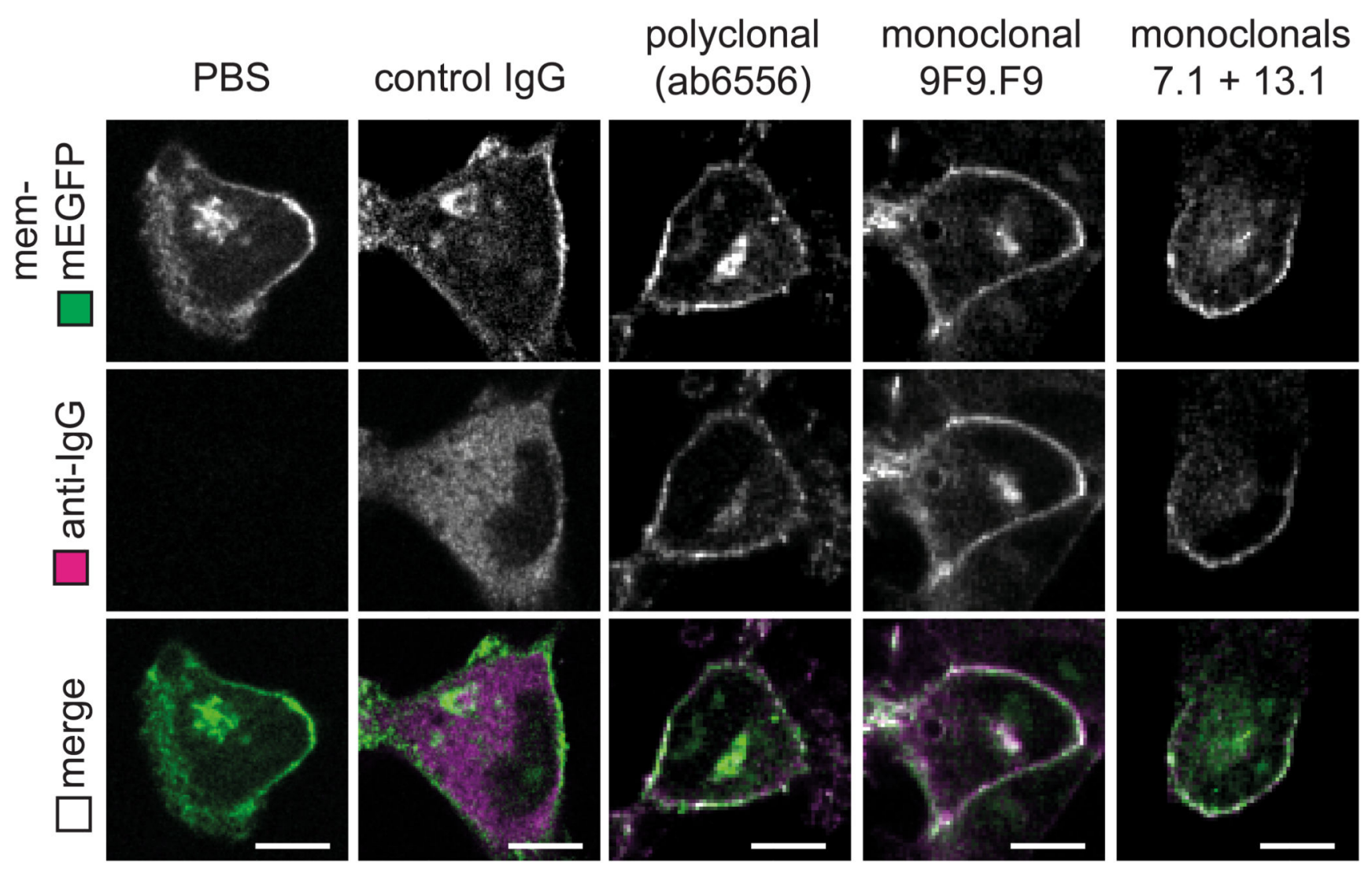

Extended Data Fig. 4. 
a

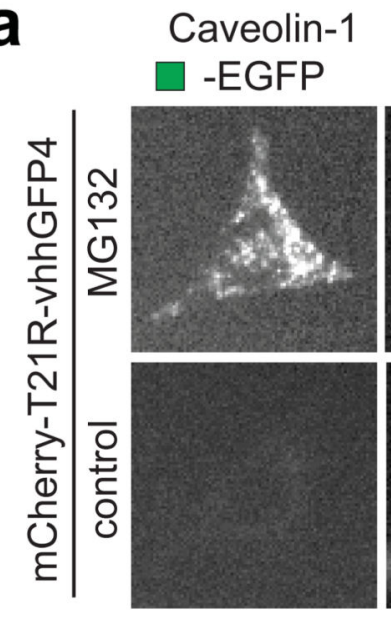

C

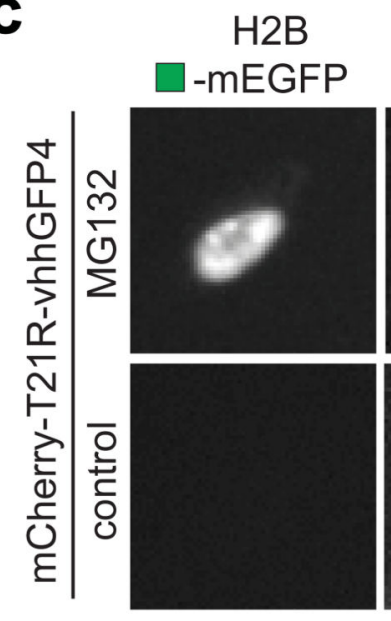

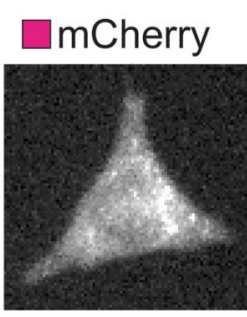
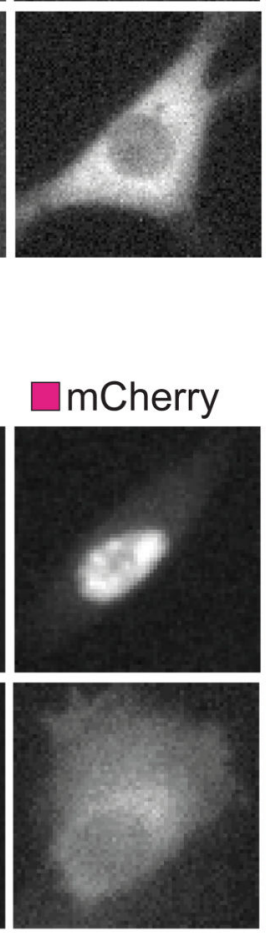
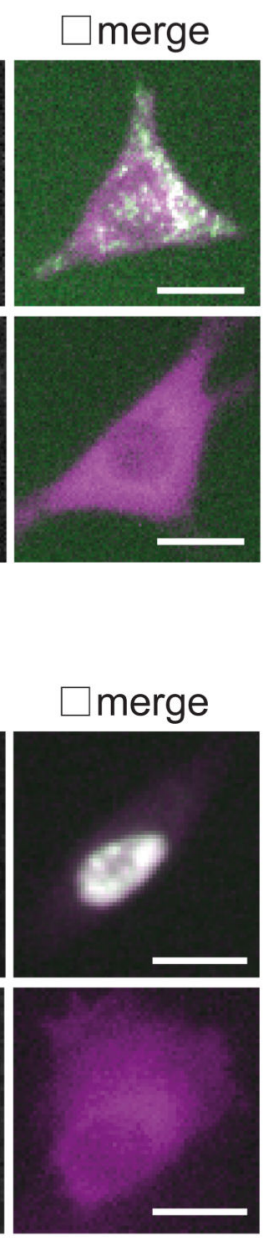

b

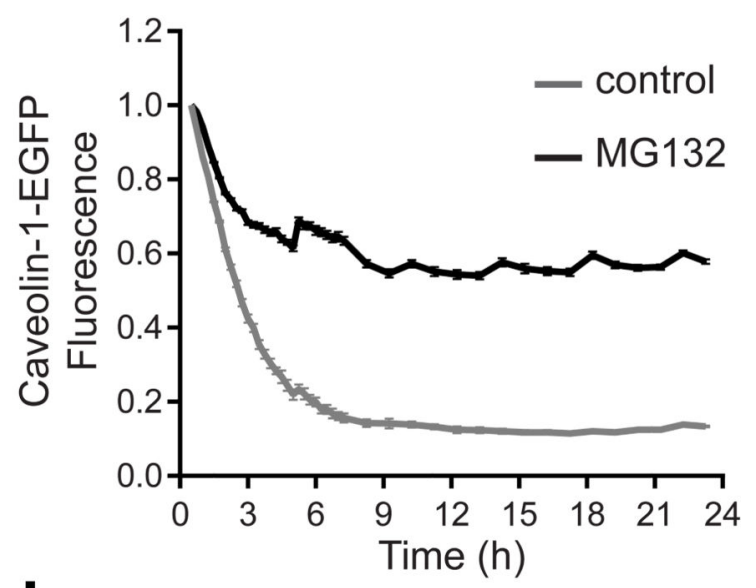

d

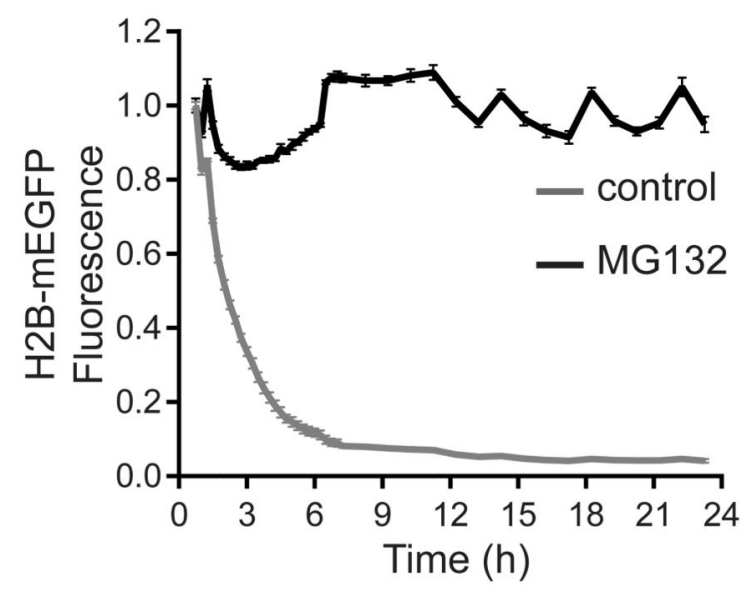

Extended Data Fig. 5.

\section{Supplementary Material}

Refer to Web version on PubMed Central for supplementary material.

\section{Acknowledgements}

We would like to thank Jan Terje Andersson (University of Oslo) for the 9C12 antibody variants and members of the James Lab for helpful discussions. J.Z. was supported by a PhD Studentship from the Rosetrees Trust and the Frank Edward Elmore Fund (University of Cambridge). M.O. is part of the GABBA PhD program from the University of Porto. D.A.J. was supported by an NHMRC Early Career Fellowship (CJ Martin) (GNT1036521). C.F.D. was supported by an NHMRC Early Career Fellowship (GNT1110116). L.K. is supported by a PhD fellowship of the Boehringer Ingelheim Fonds. W.A.M. is a Lister Institute Prize Fellow and is supported by a Sir Henry Dale Fellowship jointly funded by the Wellcome Trust and the Royal Society (Grant Number 206248/Z/17/Z) and supported by the UK Dementia Research Institute which receives its funding from UK DRI Ltd, funded by the UK Medical Research Council, Alzheimer's Society and Alzheimer's Research UK. E.M.S. acknowledges FCT - Fundação para a Ciência e a Tecnologia (contract CEECIND/00622/2017 and grant PTDC/ BEX-BCM/0432/2014) and project Norte-01-0145-FEDER-000029 supported by NORTE 2020. This work was supported by the MRC (UK; U105181010) and a Wellcome Trust Investigator Award (200594/Z/16/Z). 


\section{Data Availability}

SAXS data are available upon request. All other data generated or analysed during this study are included in this published article and associated files.

\section{References}

1. Zheng N, Shabek N. Ubiquitin Ligases: Structure, Function, and Regulation. Annu Rev Biochem. 2017; 86 :129-157. [PubMed: 28375744]

2. Schapira M, Calabrese MF, Bullock AN, Crews CM. Targeted protein degradation: expanding the toolbox. Nat Rev Drug Discov. 2019; 18 :949-963. [PubMed: 31666732]

3. Yamada T, Yang Y, Bonni A. Spatial organization of ubiquitin ligase pathways orchestrates neuronal connectivity. Trends Neurosci. 2013; 36 :218-26. [PubMed: 23332798]

4. Verma R, Mohl D, Deshaies RJ. Harnessing the Power of Proteolysis for Targeted Protein Inactivation. Mol Cell. 2020; 77 :446-460. [PubMed: 32004468]

5. Wu T, et al. Targeted protein degradation as a powerful research tool in basic biology and drug target discovery. Nature Structural \& Molecular Biology. 2020

6. James LC, Keeble AH, Khan Z, Rhodes DA, Trowsdale J. Structural basis for PRYSPRY-mediated tripartite motif (TRIM) protein function. Proc Natl Acad Sci U S A. 2007; 104 :6200-5. [PubMed: 17400754]

7. Mallery DL, et al. Antibodies mediate intracellular immunity through tripartite motif-containing 21 (TRIM21). Proceedings of the National Academy of Sciences. 2010; 107 :19985-19990.

8. Vaysburd M, et al. Intracellular antibody receptor TRIM21 prevents fatal viral infection. Proceedings of the National Academy of Sciences. 2013; $110: 12397$.

9. Fan W, et al. Swine TRIM21 restricts FMDV infection via an intracellular neutralization mechanism. Antiviral Res. 2016; 127 :32-40. [PubMed: 26777733]

10. Caddy SL, et al. Intracellular neutralisation of rotavirus by VP6-specific IgG. PLOS Pathogens. 2020; 16 e1008732 [PubMed: 32750093]

11. Caddy SL, et al. Viral nucleoprotein antibodies activate TRIM21 and induce T cell immunity. The EMBO Journal. 2020; n/a e106228

12. Rakebrandt N, Lentes S, Neumann H, James LC, Neumann-Staubitz P. Antibody- and TRIM21dependent intracellular restriction of Salmonella enterica. Pathogens and Disease. 2014; 72 :131137. [PubMed: 24920099]

13. McEwan WA, et al. Intracellular antibody-bound pathogens stimulate immune signaling via the $\mathrm{Fc}$ receptor TRIM21. Nature Immunology. 2013; 14 :327-336. [PubMed: 23455675]

14. McEwan WA, et al. Cytosolic Fc receptor TRIM21 inhibits seeded tau aggregation. Proceedings of the National Academy of Sciences. 2017201607215

15. Foss S, et al. TRIM21—From Intracellular Immunity to Therapy. Frontiers in Immunology. 2019; 10

16. McEwan WA, Mallery DL, Rhodes DA, Trowsdale J, James LC. Intracellular antibody-mediated immunity and the role of TRIM21. Bioessays. 2011; 33 :803-9. [PubMed: 22006823]

17. Clift D, et al. A Method for the Acute and Rapid Degradation of Endogenous Proteins. Cell. 2017; 171 :1692-1706. e18 [PubMed: 29153837]

18. Clift D, So C, McEwan WA, James LC, Schuh M. Acute and rapid degradation of endogenous proteins by Trim-Away. Nature Protocols. 2018; 13 :2149-2175. [PubMed: 30250286]

19. Fletcher AJ, Mallery DL, Watkinson RE, Dickson CF, James LC. Sequential ubiquitination and deubiquitination enzymes synchronize the dual sensor and effector functions of TRIM21. Proc Natl Acad Sci U S A. 2015; 112 :10014-9. [PubMed: 26150489]

20. Kiss L, et al. A tri-ionic anchor mechanism drives Ube $2 \mathrm{~N}$-specific recruitment and K63-chain ubiquitination in TRIM ligases. Nat Commun. 2019; 10 :4502. [PubMed: 31582740]

21. de Bie P, Ciechanover A. Ubiquitination of E3 ligases: self-regulation of the ubiquitin system via proteolytic and non-proteolytic mechanisms. Cell Death \& Differentiation. 2011; 18 :1393-1402. [PubMed: 21372847] 
22. Peth A, Uchiki T, Goldberg AL. ATP-Dependent Steps in the Binding of Ubiquitin Conjugates to the 26S Proteasome that Commit to Degradation. Molecular Cell. 2010; 40 :671-681. [PubMed: 21095592]

23. Hofmann RM, Pickart CM. In vitro assembly and recognition of Lys-63 polyubiquitin chains. J Biol Chem. 2001; 276 :27936-43. [PubMed: 11369780]

24. Saeki Y, et al. Lysine 63-linked polyubiquitin chain may serve as a targeting signal for the $26 \mathrm{~S}$ proteasome. EMBO J. 2009; 28 :359-71. [PubMed: 19153599]

25. Kim HT, et al. Certain pairs of ubiquitin-conjugating enzymes (E2s) and ubiquitin-protein ligases (E3s) synthesize nondegradable forked ubiquitin chains containing all possible isopeptide linkages. J Biol Chem. 2007; 282 :17375-86. [PubMed: 17426036]

26. Ohtake F, Tsuchiya H, Saeki Y, Tanaka K. K63 ubiquitylation triggers proteasomal degradation by seeding branched ubiquitin chains. Proceedings of the National Academy of Sciences. 2018; 115 E1401

27. Fletcher AJ, et al. TRIM5a requires Ube2W to anchor Lys63-linked ubiquitin chains and restrict reverse transcription. The EMBO Journal. 2015; 34 :2078-2095. [PubMed: 26101372]

28. Fletcher AJ, et al. Trivalent RING Assembly on Retroviral Capsids Activates TRIM5 Ubiquitination and Innate Immune Signaling. Cell Host \& Microbe. 2018; 24 :761-775. e6 [PubMed: 30503508]

29. Fiorentini F, Esposito D, Rittinger K. Does it take two to tango? RING domain self-association and activity in TRIM E3 ubiquitin ligases. Biochemical Society Transactions. 2020

30. Koliopoulos MG, Esposito D, Christodoulou E, Taylor IA, Rittinger K. Functional role of TRIM E3 ligase oligomerization and regulation of catalytic activity. The EMBO Journal. 2016; 35 :12041218. [PubMed: 27154206]

31. Haubrich K, et al. RNA binding regulates TRIM25-mediated RIG-I ubiquitylation. bioRxiv. 2020 2020.05.04.070177

32. Sanchez JG, et al. TRIM25 Binds RNA to Modulate Cellular Anti-viral Defense. Journal of Molecular Biology. 2018; 430 :5280-5293. [PubMed: 30342007]

33. Diaz-Griffero F, et al. Rapid turnover and polyubiquitylation of the retroviral restriction factor TRIM5. Virology. 2006; 349 :300-315. [PubMed: 16472833]

34. Li X, Sodroski J. The TRIM5a B-Box 2 Domain Promotes Cooperative Binding to the Retroviral Capsid by Mediating Higher-Order Self-Association. Journal of Virology. 2008; 82 :11495-11502. [PubMed: 18799578]

35. Yudina Z, et al. RING Dimerization Links Higher-Order Assembly of TRIM5a to Synthesis of K63-Linked Polyubiquitin. Cell Reports. 2015; 12 :788-797. [PubMed: 26212332]

36. Ganser-Pornillos BK, et al. Hexagonal assembly of a restricting TRIM5a protein. Proceedings of the National Academy of Sciences. 2011; $108: 534-539$.

37. Li Y-L, et al. Primate TRIM5 proteins form hexagonal nets on HIV-1 capsids. eLife. 2016; 5 e16269 [PubMed: 27253068]

38. Dickson $\mathrm{C}$, et al. Intracellular antibody signalling is regulated by phosphorylation of the $\mathrm{Fc}$ receptor TRIM21. eLife. 2018; 7 e32660 [PubMed: 29667579]

39. McEwan WA, et al. Regulation of Virus Neutralization and the Persistent Fraction by TRIM21. Journal of Virology. 2012; 86 :8482-8491. [PubMed: 22647693]

40. Hilpert K, et al. Anti-c-myc antibody 9E10: epitope key positions and variability characterized using peptide spot synthesis on cellulose. Protein Engineering, Design and Selection. 2001; 14 :803-806.

41. Krauss N, et al. The structure of the anti-c-myc antibody $9 \mathrm{E} 10$ Fab fragment/epitope peptide complex reveals a novel binding mode dominated by the heavy chain hypervariable loops. Proteins. 2008; 73 :552-65. [PubMed: 18473392]

42. Young G, et al. Quantitative mass imaging of single biological macromolecules. Science. 2018; 360 :423-427. [PubMed: 29700264]

43. Cole D, Young G, Weigel A, Sebesta A, Kukura P. Label-Free Single-Molecule Imaging with Numerical-Aperture-Shaped Interferometric Scattering Microscopy. ACS Photonics. 2017; 4 :211216. [PubMed: 28255572] 
44. Kubala MH, Kovtun O, Alexandrov K, Collins BM. Structural and thermodynamic analysis of the GFP:GFP-nanobody complex. Protein Science. 2010; 19 :2389-2401. [PubMed: 20945358]

45. Shvets E, Bitsikas V, Howard G, Hansen CG, Nichols BJ. Dynamic caveolae exclude bulk membrane proteins and are required for sorting of excess glycosphingolipids. Nature Communications. 2015; $6: 6867$.

46. Hayer A, Stoeber M, Bissig C, Helenius A. Biogenesis of Caveolae: Stepwise Assembly of Large Caveolin and Cavin Complexes. Traffic. 2010; 11 :361-382. [PubMed: 20070607]

47. Bates GP, et al. Huntington disease. Nature Reviews Disease Primers. 2015; 115005

48. Owens GE, New DM, West AP, Bjorkman PJ. Anti-PolyQ Antibodies Recognize a Short PolyQ Stretch in Both Normal and Mutant Huntingtin Exon 1. Journal of Molecular Biology. 2015; 427 :2507-2519. [PubMed: 26047735]

49. Bugaj LJ, Choksi AT, Mesuda CK, Kane RS, Schaffer DV. Optogenetic protein clustering and signaling activation in mammalian cells. Nature Methods. 2013; 10 :249-252. [PubMed: 23377377]

50. Taslimi A, et al. An optimized optogenetic clustering tool for probing protein interaction and function. Nature Communications. 2014; 5 :4925.

51. Park H, et al. Optogenetic protein clustering through fluorescent protein tagging and extension of CRY2. Nature Communications. 2017; $8: 30$.

52. Kono K, et al. Reconstruction of Par-dependent polarity in apolar cells reveals a dynamic process of cortical polarization. eLife. 2019; 8 e45559 [PubMed: 31172945]

53. La Spada AR, Taylor JP. Repeat expansion disease: progress and puzzles in disease pathogenesis. Nature Reviews Genetics. 2010; 11 :247-258.

54. Soto C, Pritzkow S. Protein misfolding, aggregation, and conformational strains in neurodegenerative diseases. Nature Neuroscience. 2018; 21 :1332-1340. [PubMed: 30250260]

55. Ganser-Pornillos BK, Pornillos O. Restriction of HIV-1 and other retroviruses by TRIM5. Nature Reviews Microbiology. 2019; 17 :546-556. [PubMed: 31312031]

56. Mund T, Pelham HR. Substrate clustering potently regulates the activity of WW-HECT domaincontaining ubiquitin ligases. J Biol Chem. 2018; 293 :5200-5209. [PubMed: 29463679]

57. Słabicki M, et al. Small-molecule-induced polymerization triggers degradation of BCL6. Nature. 2020

58. Zeng J, Slodkowicz G, James LC. Rare missense variants in the human cytosolic antibody receptor preserve antiviral function. eLife. 2019; 8 e48339 [PubMed: 31613747]

59. Liman ER, Tytgat J, Hess P. Subunit stoichiometry of a mammalian K+ channel determined by construction of multimeric cDNAs. Neuron. 1992; $9: 861-71$. [PubMed: 1419000]

60. Schindelin J, et al. Fiji: an open-source platform for biological-image analysis. Nat Methods. 2012; 9 :676-82. [PubMed: 22743772]

61. Schuck P. On the analysis of protein self-association by sedimentation velocity analytical ultracentrifugation. Analytical Biochemistry. 2003; 320 :104-124. [PubMed: 12895474]

62. Brautigam, CA. Methods in Enzymology. Cole, JL, editor. Vol. 562. Academic Press; 2015. 109133. 
a

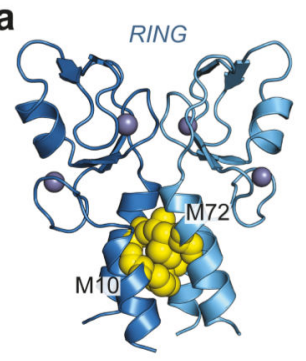

b

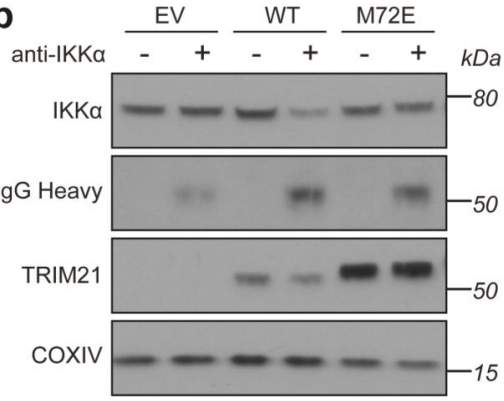

c

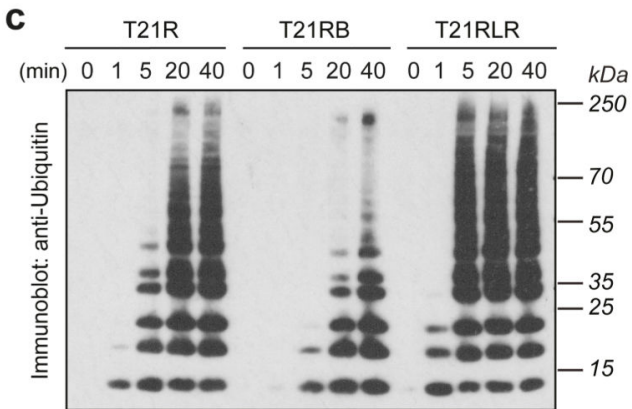

d

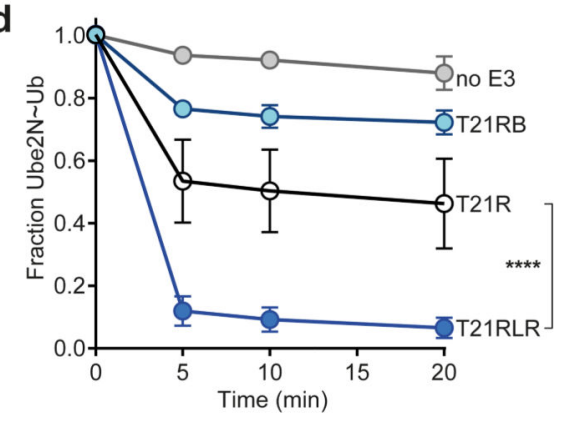

e

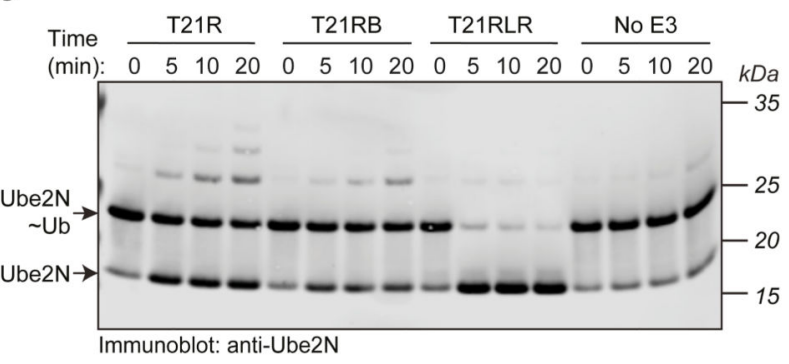

Dimeric
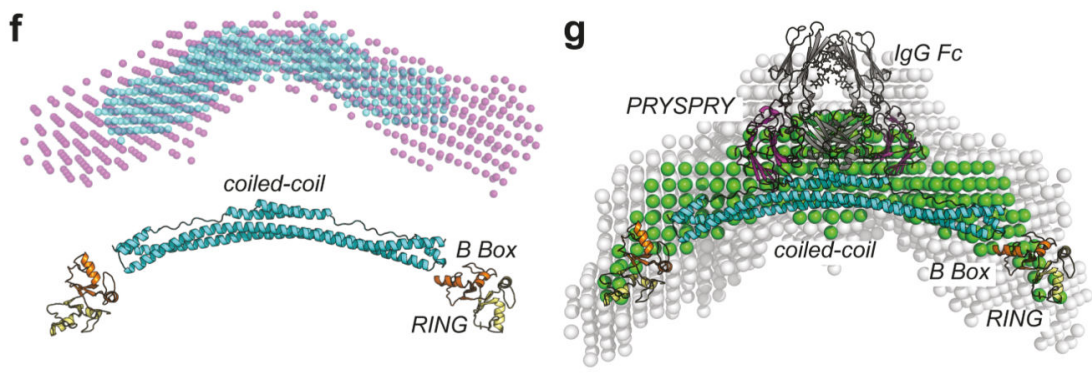

h
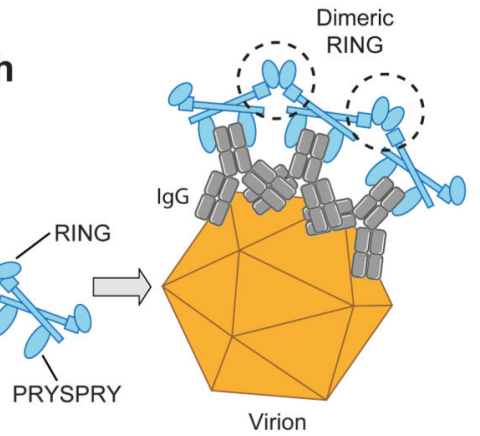

i

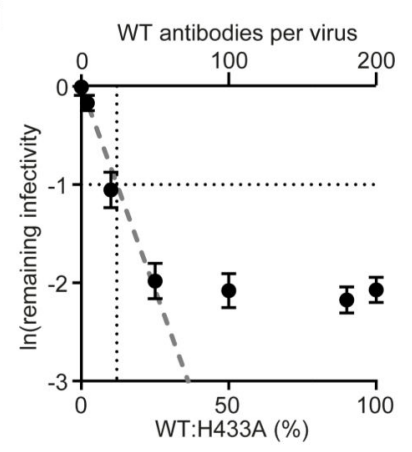

j

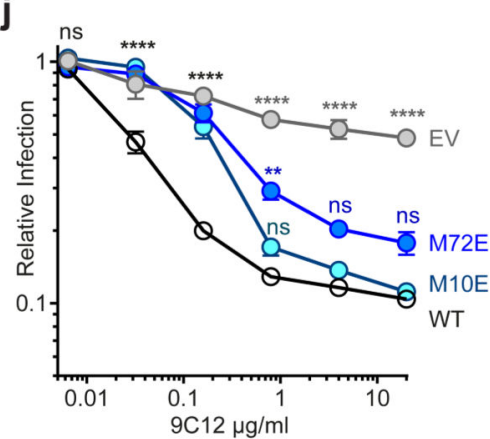

k

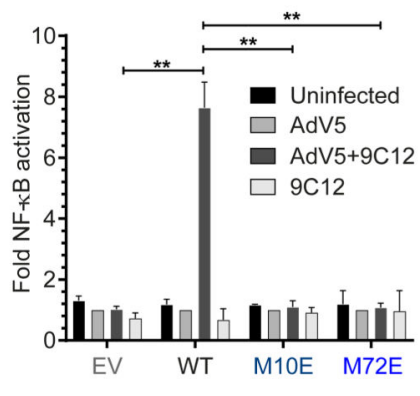

Fig. 1. RING dimerization is required for TRIM21 activation

a, Crystal structure of TRIM21 RING dimer (PDB 5OLM) ${ }^{38}$ showing the important residues M10 and M72 at the dimer interface. b, Immunoblots showing Trim-away of target protein IKKa following electroporation (+) of anti-IKKa IgG (anti-IKKa). IKKa degradation is deficient in cells expressing the M72E mutant TRIM21. c-e, T21RLR shows enhanced ubiquitination chain formation (c) and ubiquitin discharge from Ube $2 \mathrm{~N} \sim \mathrm{Ub}$ (d,e) compared to T21R. Immunoblots in b,c,e are representative examples from three independent experiments. f, Top, dummy atom model (DAM) structures calculated from 
SAXS data (Supplementary Note 1) for T21RBCC (purple spheres) and T21CC235 (blue spheres). Bottom, structural model of T21RBCC based on atomic models of the TRIM21 RING (yellow) and B Box (orange) domains (PDB 5OLM) ${ }^{38}$ and TRIM25 coiled-coil domain (cyan, PDB 4CFG). g, Averaged and filtered DAM's (white and green spheres, respectively) from TRIM21:Fc SAXS data (Supplementary Note 1) overlaid with an atomic model of the TRIM21:Fc complex using PDB 5OLM (RING, B Box), PDB 4CFG (coiledcoil) and PDB 2IWG (PRYSPRY (magenta) and IgG Fc (grey)). h, Schematic of potential intermolecular RING dimerisation on the surface of a virus-antibody complex. i, Remaining infectivity of AdV5 following incubation with saturating concentrations of varying ratios of WT:H433A 9C12 antibody. j, Neutralisation of AdV5 infection by increasing 9C12 concentrations in cells expressing the indicated TRIM21 mutants. $\mathbf{k}$, AdV5-9C12-induced NFkB activation in cells expressing the indicated TRIM21 mutants. Graphs in panels $\mathbf{d}, \mathbf{i}-\mathbf{k}$ show mean and s.e.m. for $n=3$ independent experiments; Statistical significance is based on Student's $t$-test (d), two-way ANOVA (j) and one-way ANOVA (k) and represented with labels ns (not significant, $\mathrm{P}>0.05)$ ) **(P $₫ .01)$, ****(P $₫ .0001)$, as defined in Methods. Source data for graphs and statistics are in Supplementary Data 1. Uncropped blots are shown in Supplementary Data 2. 


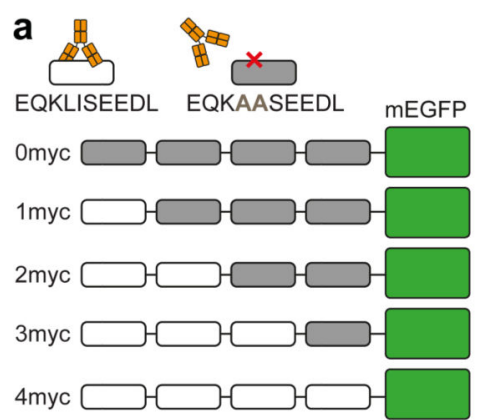

b

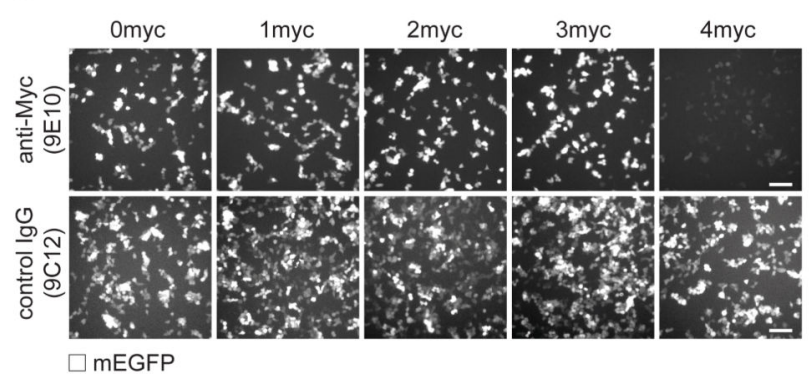

C

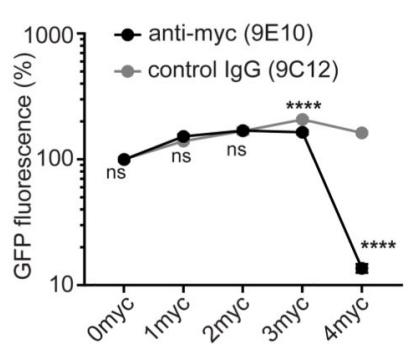

d
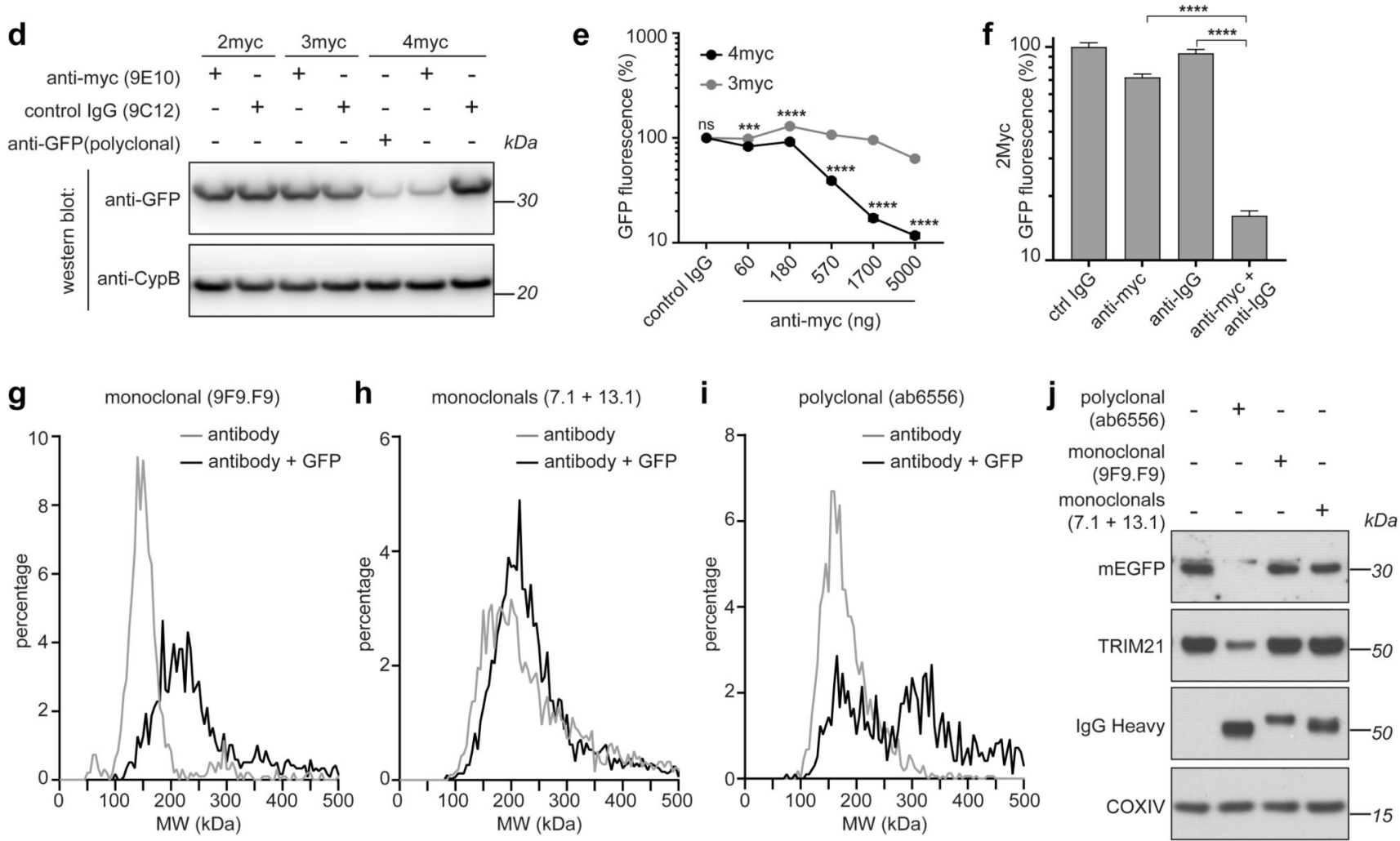

Fig. 2. Trim-Away requires recruitment of multiple TRIM21 molecules to the target.

a, Schematic of myc-mEGFP constructs. b-d, HEK293T-mCherry-TRIM21 cells were electroporated with mRNA encoding the indicated myc-mEGFP constructs together with either control IgG (9C12), ant-myc (9E10) or anti-GFP (polyclonal) antibodies. 8h postelectroporation cellular GFP fluorescence was imaged (b) and quantified (c) using the IncuCyte system, or total GFP protein levels analysed by immunoblotting (d). Scale bars, $100 \mu \mathrm{m}$. e, HEK293T-mCherry-TRIM21 cells were electroporated with mRNA encoding the indicated myc-mEGFP constructs together with control $\operatorname{IgG}$ or increasing concentrations of anti-myc antibody and GFP fluorescence quantified 8 hours later using the IncuCyte. f, HEK293T-mCherry-TRIM21 cells were electroporated with mRNA encoding 2myc-mEGFP together with the indicated antibodies and GFP fluorescence quantified 8 hours later using the IncuCyte. g--i The indicated antibodies $(50 \mathrm{nM})$ either alone, or mixed with GFP protein $(100 \mathrm{nM})$, were analysed by mass photometry. $\mathbf{j}$, RPE-1 cells expressing mEGFP were electroporated with the indicated antibodies and cell extracts immunoblotted 3 hours 
later. Incucyte images $(\mathbf{b})$, Immunoblots $(\mathbf{d}, \mathbf{j})$ and mass photometry profiles $(\mathbf{g}-\mathbf{i})$ are representative examples from three independent experiments. Graphs in c,e,f show mean and s.e.m. (some error bars are to small to be seen) from $n=3$ independent experiments. Statistical significance was calculated based on two-tailed Student's $t$-test and represented with labels ns (not significant, $\mathrm{P}>0.05$ ), *** (P $₫$ ).001), ****(P $\unlhd$ ).0001), as defined in Methods. Source data for graphs and statistics are in Supplementary Data 1. Uncropped blots are in Supplementary Data 2. 


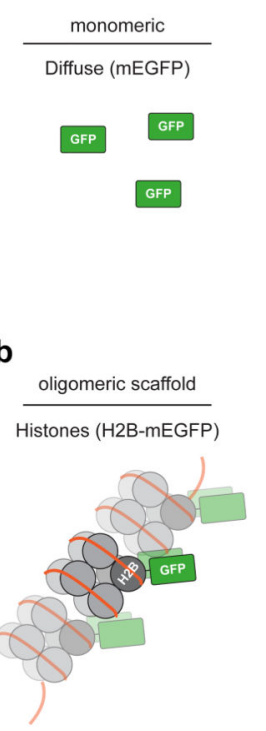

c

oligomeric scaffold

Centrosome (mEGFP-PACT)

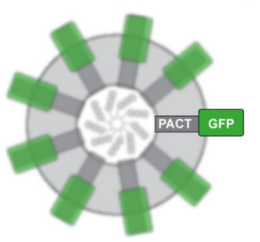

j

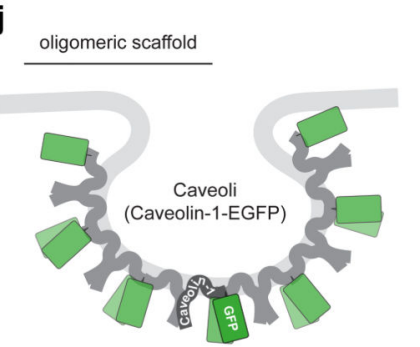

f

h

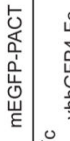
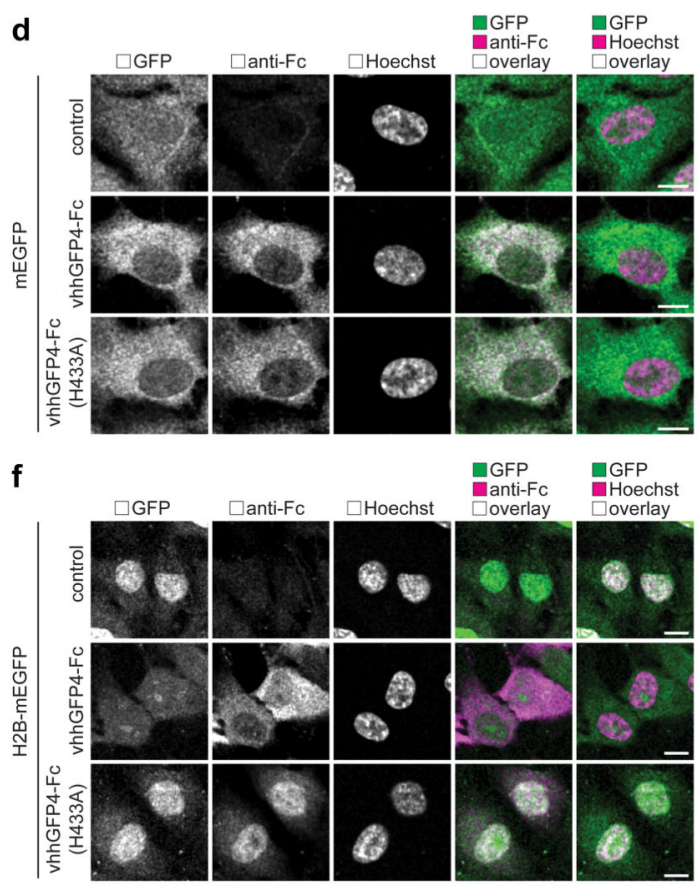

GFP
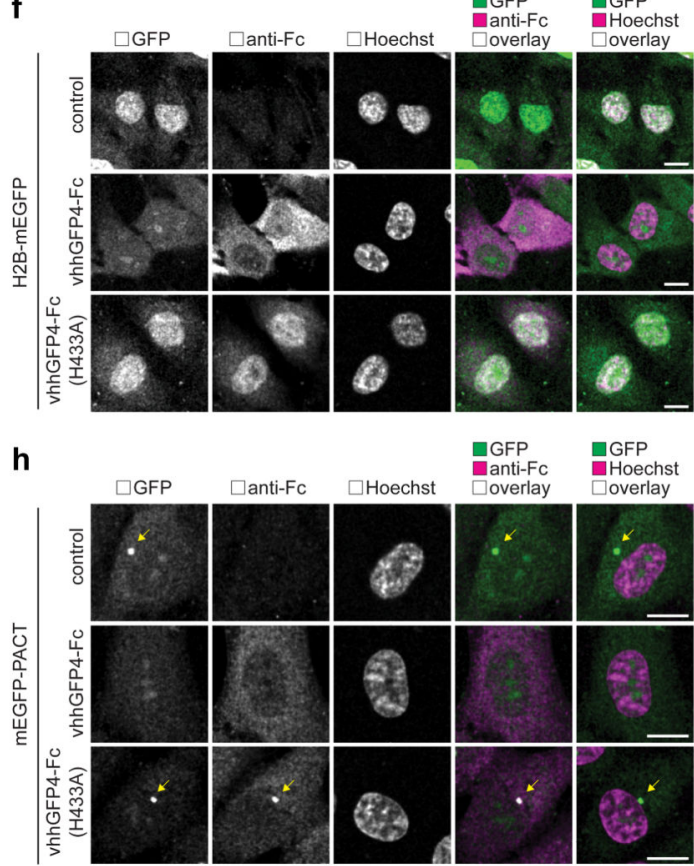

nGFP

Danti-Fc

GFP Hoechst
$\square$ overlay
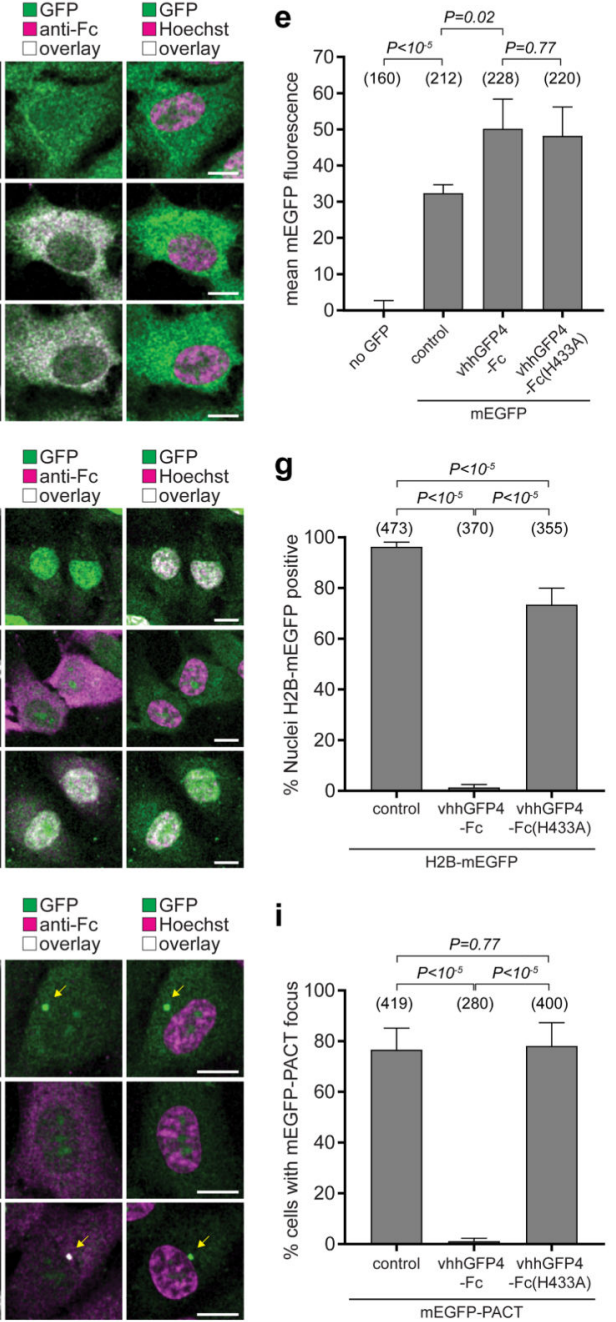

g

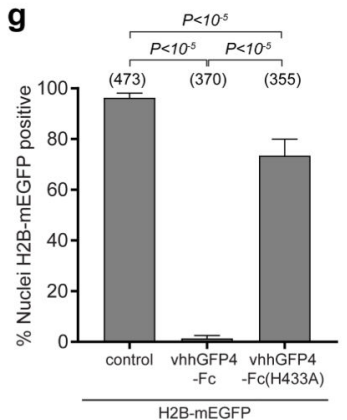

i

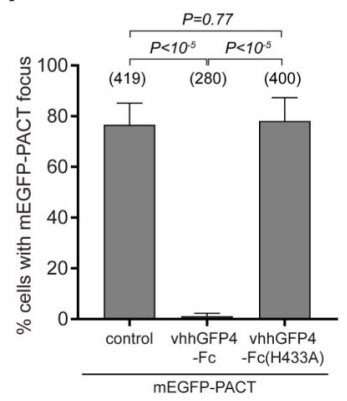

k

vhhGFP4-Fc - Caveolin-1-EGFP
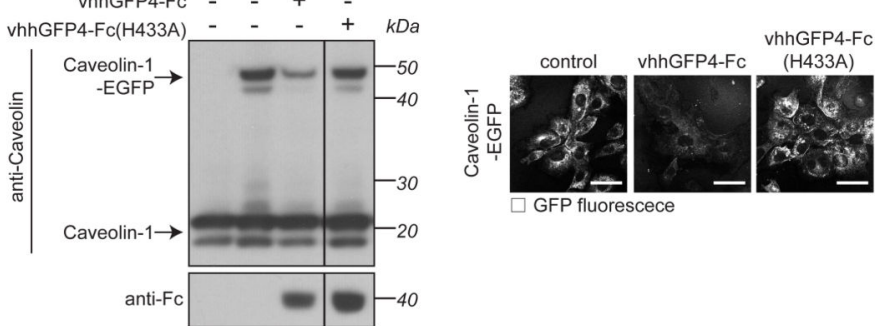

Fig. 3. The nature of the target dictates TRIM21 activation

a, mEGFP is a monomer and exhibits diffuse localisation. b,c, H2B-mEGFP and mEGFPPACT are incorporated into histones and centrosomes respectively, which act as oligomeric scaffolds for mEGFP. $\mathbf{d}-\mathbf{i}$, RPE-1 cells were electroporated with mRNA encoding mEGFP $(\mathbf{d}, \mathbf{e})$, H2B-mEGFP $(\mathbf{f}, \mathbf{g})$ or mEGFP-PACT $(\mathbf{h}, \mathbf{i})$ alone or together with vhhGFP4-Fc or vhhGFP4-Fc(H433A) and fixed 16h later for immunofluorescence. Representative confocal microscope images $(\mathbf{d}, \mathbf{f}, \mathbf{h})$ and quantification $(\mathbf{e}, \mathbf{g}, \mathbf{i})$ show that $\mathrm{mEGFP}$ is degraded in a TRIM21-dependent manner by vhhGFP4-Fc only when tethered to the oligomeric histone 
and centrosome scaffolds. Scale bars, $10 \mu \mathrm{m}$. Yellow arrows in $\mathbf{h}$ show centrosomes. Graphs in e,g,i show mean and s.d. from $n=3$ independent experiments. Number of cells quantified in brackets. P values from two-tailed Student's $t$-test. j, Schematic of mEGFP fused to oligomeric Caveolin-1. k,i, WT NIH3T3 or NIH3T3-Caveolin-1-GFP cells were electroporated with mRNA encoding vhhGFP4-Fc or vhhGFP4-Fc(H433A) and 16 hours later analysed by immunoblotting (k) and confocal microscopy (l). Scale bars, $50 \mu \mathrm{m}$. Source data for graphs and statistics are in Supplementary Data 1. 
a

Human Huntingtin protein
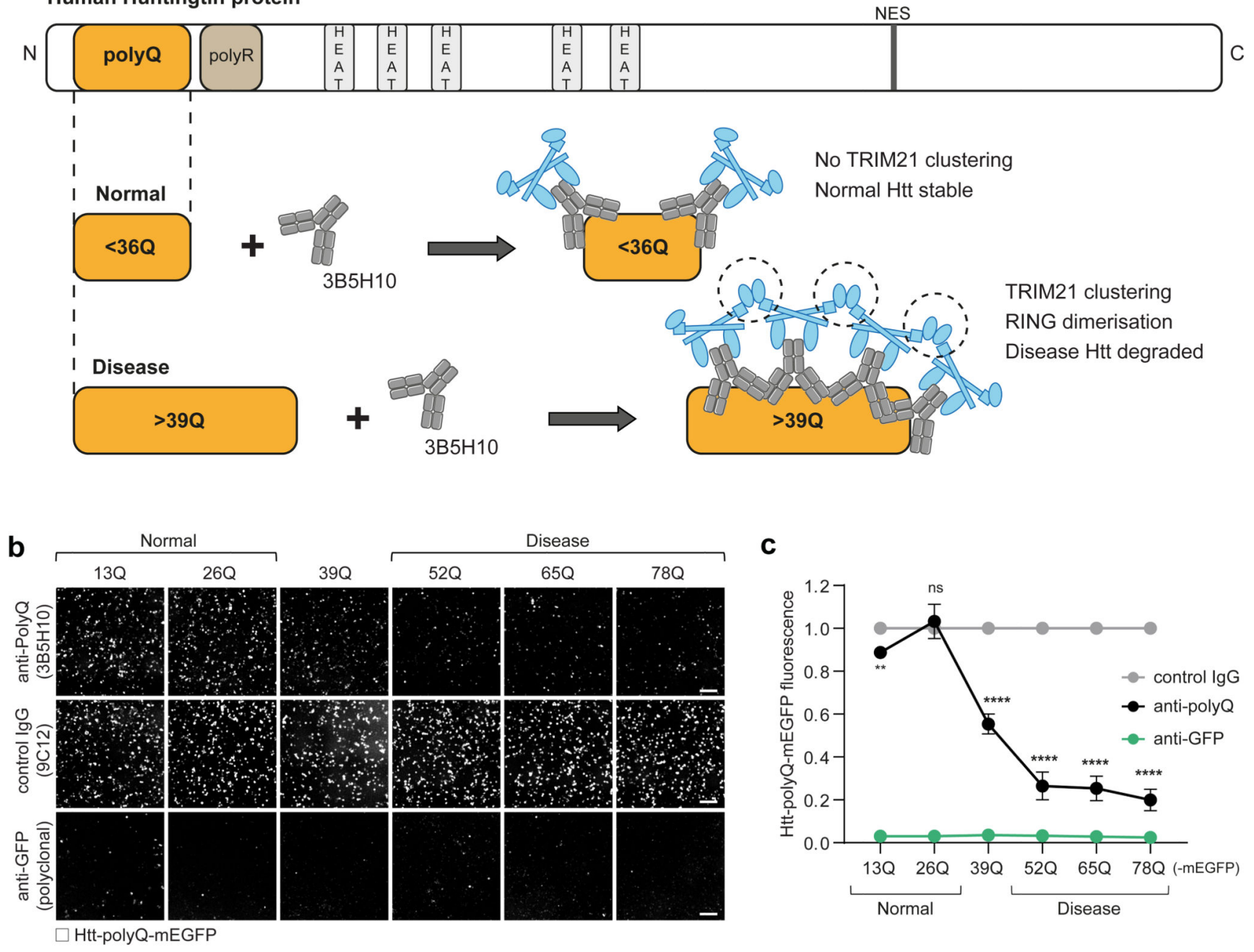

Fig. 4. Selective degradation of disease-causing huntingtin by TRIM21

a, Schematic of human huntingtin protein highlighting the $\mathrm{N}$-terminal polyglutamine (polyQ) tract. PolyQ tracts greater than 39Q are associated with Huntington's disease. The anti-polyQ monoclonal antibody 3B5H10 binds to a 13Q epitope. Up to two 3B5H10 antibodies are predicted to bind to normal huntingtin, with additional antibodies predicted to bind as the length of polyQ increases, which would lead to TRIM21 clustering, activation and degradation of only huntingtin protein with long, disease-causing, polyQ tracts. b,c, HEK293T-mCherry-TRIM21 were electroporated with mRNA encoding the indicated polyQ-mEGFP constructs together with either anti-polyQ (3B5H10), control IgG (9C12) or anti-GFP (polyclonal) antibodies. 8 hours post-electroporation cellular GFP fluorescence was imaged (b) and quantified (c) using the IncuCyte system. Scale bars 100 $\mu \mathrm{m}$. Data points in c show mean and s.e.m. (some error bars are too small to be seen), $n=3$ independent experiments. Statistical significance is based on two-tailed Student's $t$-test and

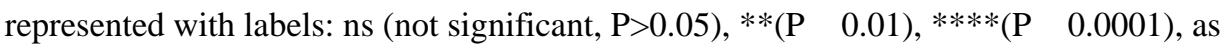
defined in Methods. Source data for graphs and statistics are in Supplementary Data 1. 
a

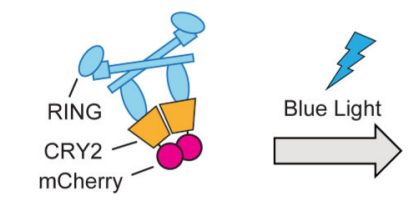

TRIM21-CRY2-mCherry
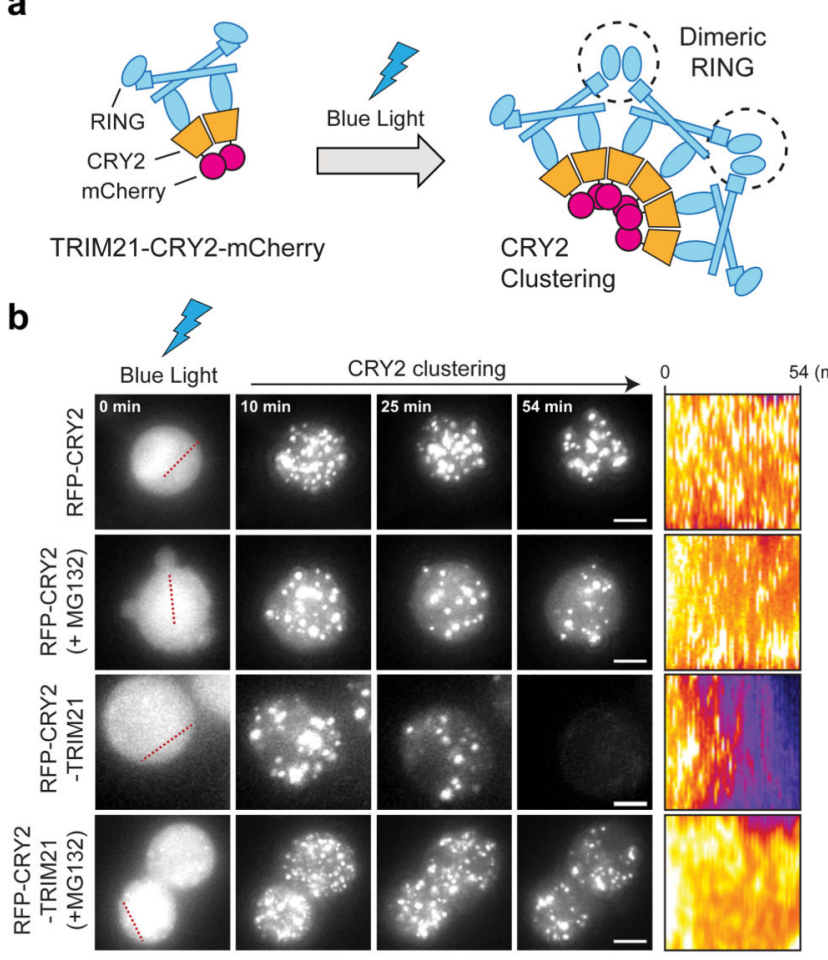

C

$54(\mathrm{~min})$

d

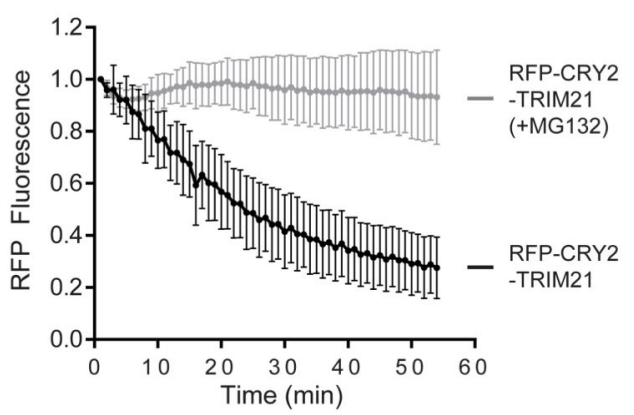

d $\quad$ TRIM21- T21(E13A)- T21(M10E/M72E)-

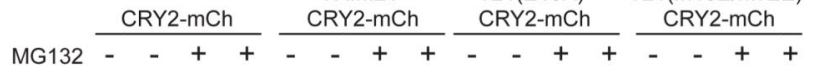

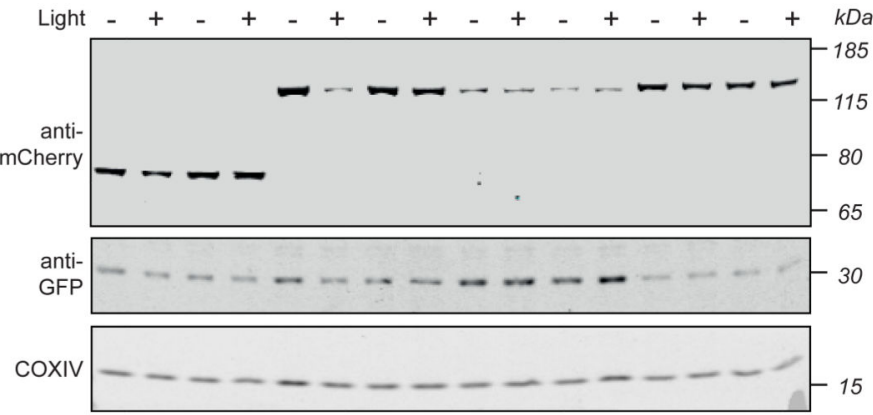

Fig. 5. Molecular clustering activates TRIM21

a, Schematic of light-induced clustering of TRIM21. b,c, Drosophila S2 cells expressing the indicated constructs were incubated with or without MG132 and RFP fluorescence quantified by live imaging. Time shows minutes ( $\mathrm{min}$ ) from onset of blue light exposure. Scale bars, $5 \mu \mathrm{m}$. Pseudo-coloured kymographs show fluorescence intensity in regions defined by red dotted lines. Graph shows mean and s.d. of fluorescence intensity of RFPCRY2-TRIM21 ( $\mathrm{n}=23$ cells) and RFP-CRY2-TRIM21+MG132 ( $\mathrm{n}=20$ cells) normalised for the respective controls (RFP-CRY2 ( $\mathrm{n}=16$ cells) and RFP-CRY2 + MG132 ( $\mathrm{n}=15$ cells). d, RPE-1 cells expressing the indicated constructs together with mem-mEGFP were incubated with or without MG132 and blue light for 3 hours prior to immunoblotting. Representative immunoblots from three independent experiments. Source data for graphs are Supplementary Data 1. Uncropped blots are in Supplementary Data 2. 
optoTrim-Away construct

$\underbrace{\text { Nanobody }}_{\begin{array}{c}\text { Target Binding } \\ \text { (vhhGFP4) }\end{array}} \underbrace{\text { RFP }}_{\text {Reporter }} \underbrace{\text { CRY2 }}_{\begin{array}{c}\text { Light-induced } \\ \text { clustering }\end{array}} \underbrace{\text { TRIM21 }}_{\begin{array}{c}\text { E3 Ligase activated } \\ \text { by clustering }\end{array}}$

b

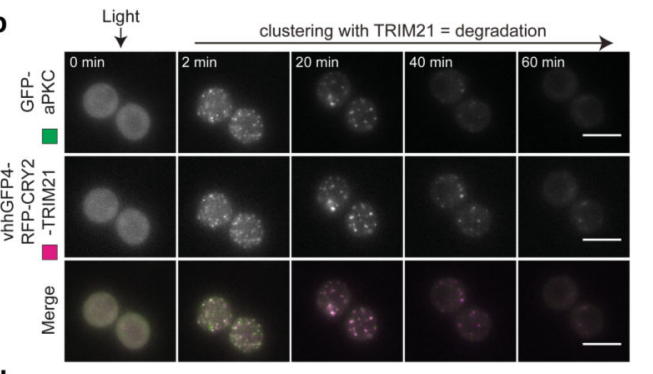

C

d

clustering without TRIM21 = no degradation

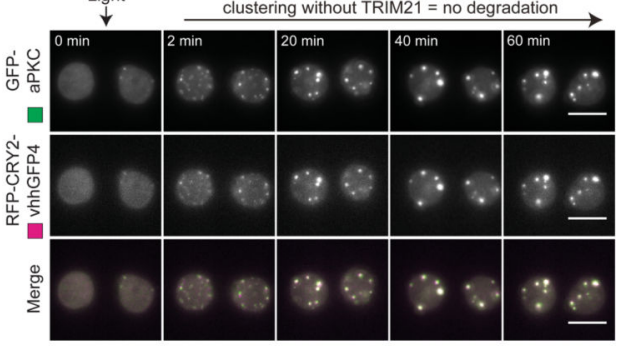

$1.27 \rightarrow$ GFP-aPKC

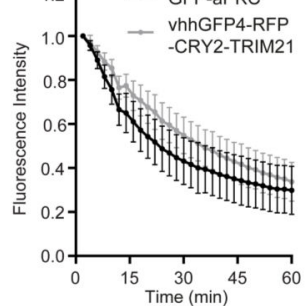

e

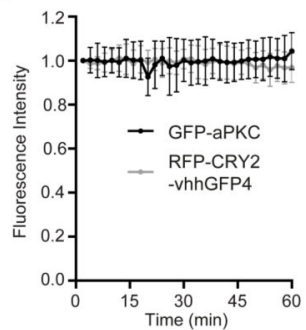

f
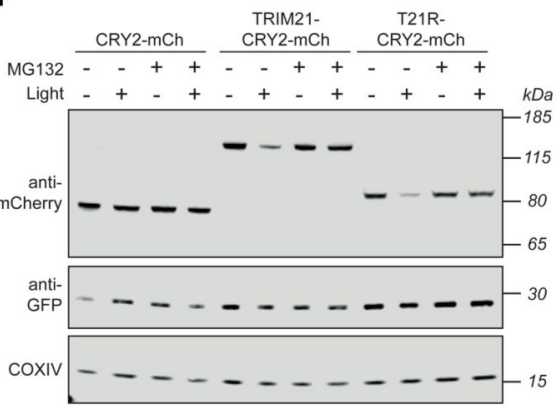

g miniTrim-Away construct

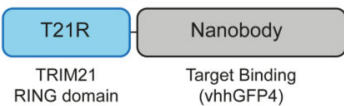

h cell NiNTA size exclusion
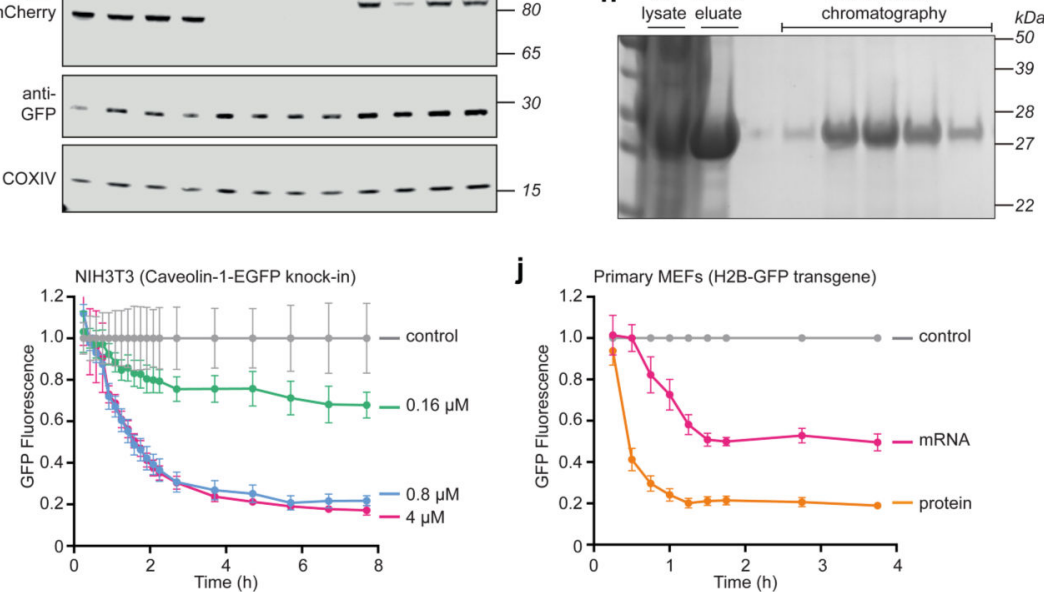

j

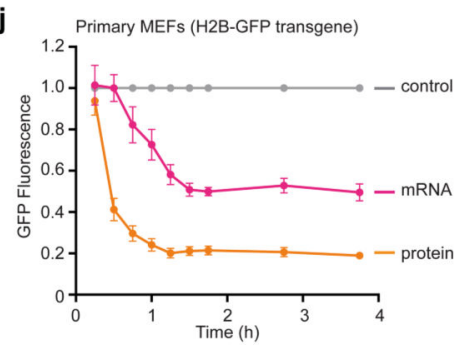

Fig. 6. New ways to Trim-Away proteins

a, Schematic of optoTrim-Away construct. b-e, Drosophila S2 cells expressing GFP-aPKC and vhhGFP4-RFP-CRY2-TRIM21 (b,c) or RFP-CRY2-vhhGFP4 (d,e) were analysed by live imaging. Time shows minutes ( $\mathrm{min}$ ) from onset of blue light exposure. Scale bars, $10 \mu \mathrm{m}$. Graphs show mean and s.d. of GFP and RFP fluorescence intensity $(\mathrm{n}=30$ cells in $\mathbf{c}$ and $n=24$ cells in $\mathbf{e}$ ). $\mathbf{f}$, RPE- 1 cells expressing the indicated constructs together with mem-mEGFP were incubated with or without MG132 and blue light for 3 hours prior to immunoblotting. $\mathbf{g}$, Schematic of miniTrim-Away construct. $\mathbf{h}$, Bacterially-expressed 
6His-T21R-vhhGFP4 was purified using a two-step protocol of NiNTA- followed by size exclusion- chromatography and analysed by Coomassie blue staining of SDS-PAGE. i, NIH3T3-Caveolin-1-GFP cells were electroporated with PBS (control) or the indicated concentrations of T21R-vhhGFP4 protein and GFP fluorescence was quantified using the IncuCyte system. j, H2B-GFP primary MEFs were electroporated with PBS (control) or T21R-vhhGFP4 in the form or mRNA or protein and GFP fluorescence quantified using the IncuCyte system. Graphs in $\mathbf{i}$ and $\mathbf{j}$ show mean and s.e.m. of $n=4$ technical replicates. Representative immunoblots (f) and GFP degradation profiles (i,j, from three independent experiments. Source data for graphs are Supplementary Data 1. Uncropped blots are in Supplementary Data 2. 


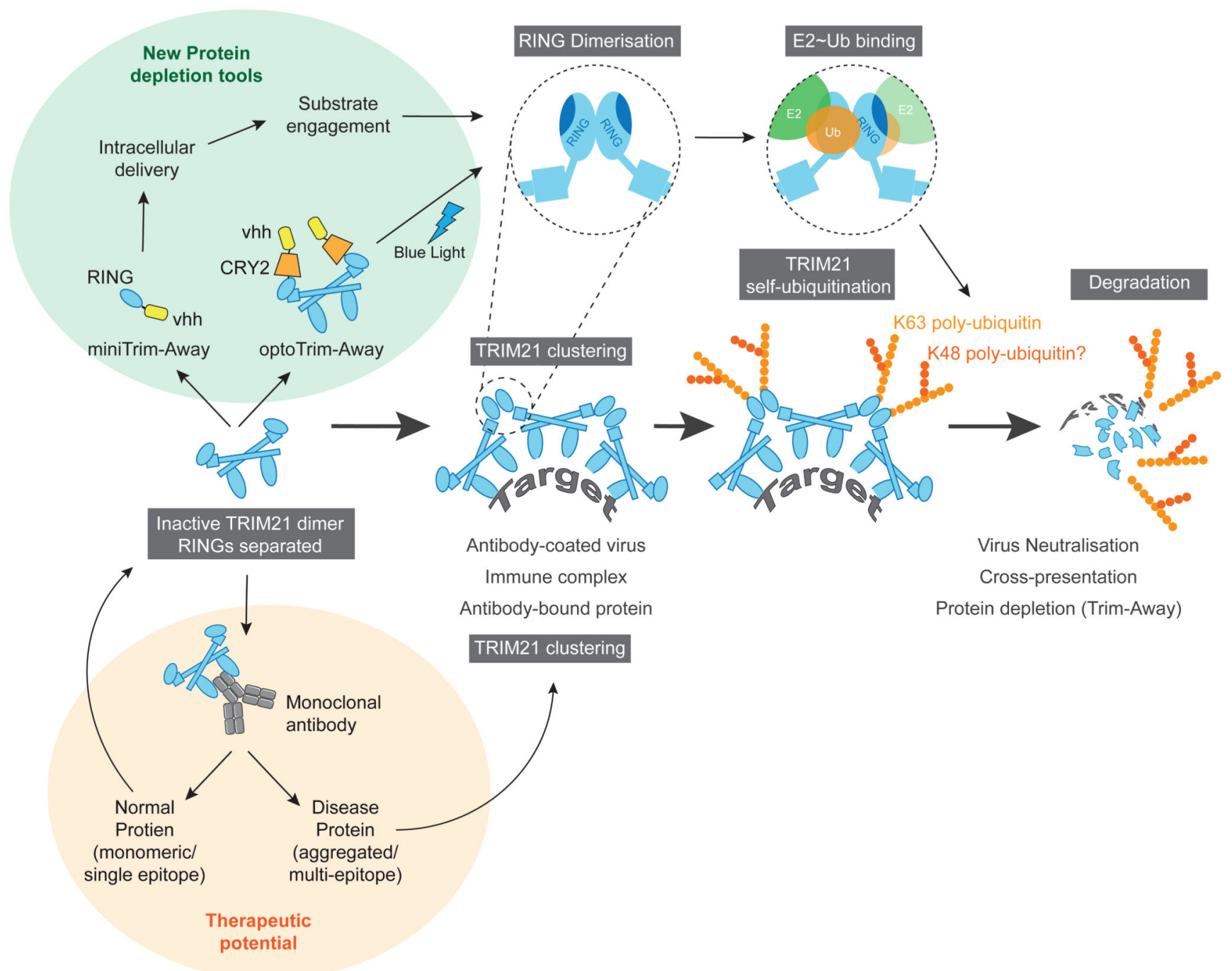

Fig. 7. Model for target-induced clustering mechanism and its applications The two RING domains of the TRIM21 dimer are held apart by an elongated antiparallel coiled-coil. Target-induced clustering allows intermolecular RING dimerisation, which facilitates E2 Ub binding and catalysis of K63-linked ubiquitin chains on TRIM21 leading to proteasomal degradation of TRIM21 and its targets. TRIM21 clustering can be induced by antibody-coated virus, internalised immune complexes or proteins targeted with antibodies. This mechanism has therapeutic potential for selective degradation of repeat-epitope or aggregated disease proteins (orange shaded area). New protein depletion tools based on the sufficiency of molecular clustering and RING dimerisation for TRIM21-mediated degradation (green shaded area). 